\title{
Association between vitamin $D$ receptor (VDR) polymorphisms and the risk of multiple sclerosis (MS): an updated meta- analysis
}

Danyal Imani ${ }^{1}$, Bahman Razi ${ }^{2}$, Morteza Motallebnezhad ${ }^{3,4,5}$ and Ramazan Rezaei ${ }^{6^{*}}$ (1)

\begin{abstract}
Background: The association between the Vitamin D Receptor (VDR) gene polymorphism and the risk of Multiple sclerosis (MS) has been evaluated in several researches. However, the findings were inconsistent and inconclusive. Therefore, we set out a meta-analysis of all eligible published case-control studies to obtain an exact evaluation of the association between VDR gene polymorphisms and MS.

Method: All relevant studies reporting the association between the VDR gene Fokl (rs2228570), or/and Taql (rs731236) or/ and Bsml (rs1544410) or/and Apal (rs7975232) polymorphisms and susceptibility to MS published up to May, 2019 were identified by comprehensive systematic search in the electronic database of web of science, Scopus, and PubMed. After that, the strength of association between VDR gene polymorphisms and susceptibility to MS was evaluated by odds ratio (OR) and $95 \%$ confidence interval (Cl).

Results: A total of 30 case-control studies were included in the meta-analysis. The overall results suggested a significant association between Taql polymorphism and MS risk under heterozygote genetic model $(\mathrm{OR}=1.27,95 \% \mathrm{Cl}=1.01-1.59$, random effect). Moreover, the pooled results of subgroup analysis declined presence of significant association under all defined genetic model. In subgroup analysis, Bsml polymorphisms was associated with increased risk of MS under recessive model in Asian populations. On the other hand, Apal polymorphism was associated with decreased risk of MS under recessive and aa vs. AA model in Asian populations.

Conclusion: This meta-analysis suggested a significant association between Taql polymorphism and MS susceptibility. Furthermore, Bsml polymorphism was associated with increased risk of MS in Asian populations. In contrast, Apal polymorphism was associated with decreased risk of MS in Asian populations. Future large-scale studies on gene-environment and gene-gene interactions are required to estimate risk factors and assist early diagnosis of patients at high risk for MS.
\end{abstract}

Keywords: Vitamin D receptor, Multiple sclerosis, Polymorphism, Meta-analysis

\footnotetext{
* Correspondence: ramin.rezaei25@gmail.com

${ }^{6}$ Department of Immunology, School of Medicine, Shahid Beheshti University

of Medical Sciences, Tehran 14194, Iran

Full list of author information is available at the end of the article
}

(c) The Author(s). 2019 Open Access This article is distributed under the terms of the Creative Commons Attribution 4.0 International License (http://creativecommons.org/licenses/by/4.0/), which permits unrestricted use, distribution, and reproduction in any medium, provided you give appropriate credit to the original author(s) and the source, provide a link to the Creative Commons license, and indicate if changes were made. The Creative Commons Public Domain Dedication waiver (http://creativecommons.org/publicdomain/zero/1.0/) applies to the data made available in this article, unless otherwise stated. 


\section{Background}

Multiple sclerosis (MS) is a chronic and demyelinating disorder of the brain and spinal cord that mainly develops in young individuals $[1,2]$. Tissue damage in MS results from a dynamic and complex interaction between the glia (oligodendrocytes and their precursors, astrocytes, and microglia), neurons, and immune system. Although there is an argument about whether the original cause of MS is extrinsic or intrinsic to the CNS, several researches in animal models in combination with evaluation of immune cells in humans have elucidated a fundamental function for adaptive immunity $[3,4]$. It has been demonstrated that genetic and environmental factors play important roles in susceptibility to the disease [5]. Vitamin D is a group of fat-soluble secosteroids that have functional and regulatory effects in the body. Vitamin D has been implicated in the development of the brain and spinal cord. Alternatively, the active form of vitamin D, 1,25-dihydroxyvitamin $\mathrm{D}$ has a wide anti-inflammatory and immunomodulatory properties $[6,7]$. Vitamin D exerts its immunomodulatory functions within the immune system by decreasing the presentation of major histocompatibility complex (MHC) II on T cells and monocytes. Vitamin D also reduces $\mathrm{T}$ cell proliferation and pro-inflammatory cytokine release [8]. The lower serum vitamin D levels compared to healthy controls have been reported in MS patients. Moreover, Vitamin D has positive effects in regulating MS risk development $[9,10]$. The effects of Vitamin D on the immune system are exerted by binding to the nuclear Vitamin D Receptor (VDR) [11]. Particular variants of the VDR gene are related to changes in vitamin $\mathrm{D}$ metabolism and function [12]. Taken together, these results suggested that VDR may play an important role in the pathogenesis of MS.

The human VDR gene is located on the chromosome $12 \mathrm{q} 12-14$ and series of restriction fragment length polymorphisms (RFLP) in the human VDR gene have been reported, containing BsmI (rs1544410), ApaI (rs7975232), FokI (rs2228570), and TaqI (rs731236) restriction sites [13]. ApaI, BsmI, and TaqI are localized near the 3 '-untranslated region (UTR) of the $V D R$ gene in the intron between exons 8 and 9, and shown to be in strong linkage disequilibrium (LD) with each other [14]. The 3'-UTR of the VDR gene is involved in the regulation of gene expression by regulating the mRNA stability and expression level [15]. Polymorphism FokI is located at the translation starting codon [16].

The association between MS and VDR gene single nucleotide polymorphisms (SNPs) has been investigated in several studies. Particularly, studies have evaluated associations between the most common SNPs of the VDR gene (TaqI, ApaI, FokI, and BsmI polymorphisms) and MS. While studies in Australia [17], Kuwait [18], and southeast of Iran [19] reported a significant association between TaqI, ApaI, and FokI polymorphisms and MS, other studies in Tunisia [20], Slovakia [21], and Greece [22] have failed to find such association. The reasons for this disparity may be small sample sizes, low statistical power, clinical heterogeneity, or a combination of these factors. To offset these limitations, this meta-analysis was performed to investigate whether VDR gene polymorphisms contribute to MS or not. Up to now, there are four meta-analysis which investigated the association between VDR polymorphisms and MS. The two studies performed by Huang et al. [23]., and Garcia-Martin et al. [24]. have indicated that there was no association between $V D R$ gene polymorphism and MS risk. Nevertheless, the other meta-analysis by Zhang et al. [25]., and Tizaoui et al. [26]. demonstrated a significant association between ApaI and FokI and MS susceptibility. Since publishing of the last meta-analysis, seven new studies have been founded in electronic databases. Therefore, we conducted a meta-analysis of all eligible published case-control studies to obtain an exact evaluation of the association between VDR gene polymorphisms and susceptibility to MS.

\section{Methods}

The current systematic review and meta-analysis was conducted according to the Preferred Reporting Items for Systematic Reviews and Meta-Analyses (PRISMA) statement [27].

\section{Publication search}

All relevant studies reporting the association between the VDR gene FokI (rs2228570) or/and TaqI (rs731236) or/and BsmI (rs1544410) or/and ApaI (rs7975232) polymorphisms and susceptibility to MS published up to May 2019 were identified by comprehensive systematic search in the electronic database of web of science, Scopus, and PubMed. The following search terms were applied: (VDR" or "vitamin D receptor") AND ("multiple sclerosis" OR "MS") AND ("polymorphisms" OR "single nucleotide" OR "polymorphism" OR "SNP" OR "variation" OR "mutation"). As a complementary approach, in order to detect additional potentially relevant studies, manual evaluation of the reference list of the included eligible studies was performed. In this meta-analysis, the strategy of search was restricted solely to the Englishlanguage publications and human population.

\section{Study selection}

Two reviewers independently assessed titles and abstract of all studies retrieved in the initial search. Articles not following the eligibility criteria were excluded by applying a hierarchical approach based on study design. Fulltext examination was applied if we could not decide include or exclude based on titles and abstract. In particular conditions, if an author has published more than one study by the same case series, the most recently published study was included. Any disagreements were discussed and resolved by consensus. 


\section{Eligibility criteria}

Studies considered eligible if meet the following criteria: 1) All eligible case-control studies that evaluate the relationship between the VDR gene single nucleotide polymorphisms and the risk of MS as the main outcome; 2) Sufficient data are available to extract or calculate odds ratios (ORs) and 95\% confidence intervals (CI); 3) Contained genotypic or allelic distributions of case and healthy individuals for $V D R$ gene polymorphism in the studies. The exclusion criteria were as follows: 1) Studies which genotype or allelic frequency could not be extracted; 2) Letters, case reports, reviews, comments, book chapter, and abstracts; 3) Duplicated reports and studies with repetitive subjects. The application of these criteria yielded 30 case-control studies eligible for the meta-analysis.

\section{Data extraction}

Two reviewers independently extracted all data according to standardized extraction form for the following data: The author's name, journal and year of publication, country of origin, ethnicity, number of cases and controls for every gender separately, mean or range of age, genotyping method, total sample size of cases and controls, and the number of cases and controls for each genotype. For quality assessment of the included publications, the Newcastle-Ottawa Scale (NOS) was applied [28]. Studies with scores $0-3,4-6$ or 7-9 were of low, moderate or high-quality, respectively.

\section{Statistical analysis}

Deviation from Hardy-Weinberg equilibrium (HWE) for distribution of the allele frequencies was analyzed using Chi-Square test in control group. Sensitivity analysis was conducted to estimate the stability of the results by removing the studies not in HWE. The strength of association between the VDR gene FokI, TaqI, BsmI, and ApaI polymorphisms and susceptibility to MS was evaluated by OR and 95\% CI. Defined model for FokI, TaqI, BsmI, ApaI were as follow, respectively: FokI; dominant model $(\mathrm{ff}+\mathrm{Ff}$ vs. FF), recessive model ( $\mathrm{ff}$ vs. $\mathrm{Ff}+\mathrm{FF})$, allelic model (f vs. F), homozygote model (ff vs. FF), and heterozygote model (Ff vs. FF); TaqI; dominant model ( $\mathrm{tt}+$ Tt vs. TT), recessive model ( $t \mathrm{t}$ vs. $\mathrm{Tt}+\mathrm{TT}$ ), allelic model ( $\mathrm{t}$ vs. T), homozygote model (tt vs. TT), and heterozygote model (Tt vs. TT); BsmI; dominant model (bb $+\mathrm{Bb}$ vs. $\mathrm{BB})$, recessive model (bb vs. $\mathrm{Bb}+\mathrm{BB})$, allelic model ( $\mathrm{b}$ vs. $\mathrm{B}$ ), homozygote model (bb vs. $\mathrm{BB}$ ), and heterozygote model (Bb vs. BB); ApaI; dominant model (aa+Aa vs. $\mathrm{AA}$ ), recessive model (aa vs. Aa+AA), allelic model (a vs. A), homozygote model (aa vs. AA), and heterozygote model (Aa vs. AA). For each genetic model, subgroup analysis was applied to evaluate ethnicity effects. In consideration of the possibility of heterogeneity (between study variability) across included studies, chi-square based Q-test was used [29]. Additionally, to show possible heterogeneity quantitatively, the other index $\left(I^{2}\right)$ was calculated as the percentage of heterogeneity. There was significant heterogeneity if an $I^{2}$ values exceeded $50 \%$ or the $Q$ statistic had a $P$ value less than 0.1 . In the presence of significant heterogeneity, the random effects model (DerSimonian-Laird approach) was performed. Otherwise, the fixed effects model (Mantel-Haenszel approach) was performed for combination of data [30, 31]. Visual inspection of asymmetry in funnel plots asymmetry, Begg's test, and Egger's test were conducted to evaluate publication bias $(P$ value $<0.05$ was considered statistically significant) [32, 33]. The data analyses were carried out using STATA (version 14.0; Stata Corporation, College Station, TX) and SPSS (version 23.0; SPSS, Inc. Chicago, IL).

\section{Results}

\section{Study characteristics}

The primary search in web of science, Scopus, and PubMed databases yielded a total of 636 publications. After removal of duplicates and evaluation of title/abstract, only 76 studies remained for full-text examination. Eventually, 30 studies met the inclusion criteria and included for quantitative synthesis. The search workflow is shown in Fig. 1. Study characteristics are summarized in Table 1. Among 30 eligible studies, 16 Studies investigated FokI SNP, 23 Studies TaqI SNP, 16 studies BsmI SNP and 20 Studies ApaI SNP. The studies were published between 1999 and 2019. Taq-Man and PCR-RFLP genotyping methods were used by the most studies.

\section{Quantitative synthesis}

The distributions of FokI, TaqI, BsmI and ApaI genotypes of the included studies are shown in Table 2. FF for FokI SNP, TT for TaqI SNP, BB for BsmI SNP and AA for ApaI were used as the reference category. The heterogeneities in the comparisons $\left(I^{2}<50 \%\right.$, fixedeffects models; $I^{2}>50 \%$, random-effects models) ascertained the application of Fixed-effects or random-effects models.

Meta-analysis for Fokl (rs2228570) polymorphism and MS Overall 16 case-control studies with 3057 cases and 2852 controls were analyzed for assessment of FokI gene polymorphism and MS risk. Of 16 studies, 9 studies carried out in Europe continent [21, 24, 34-40] 4 studies in Asia continent $[18,19,41,42]$ one study in America continent [43] and finally 2 studies in Australia [17, 44] (Table 1). No significant association was observed between FokI polymorphism and MS risk across all genetic models. Additionally, subgroup analysis based on geographical location was performed which the pooled results rejected any association between FokI polymorphism and risk of 


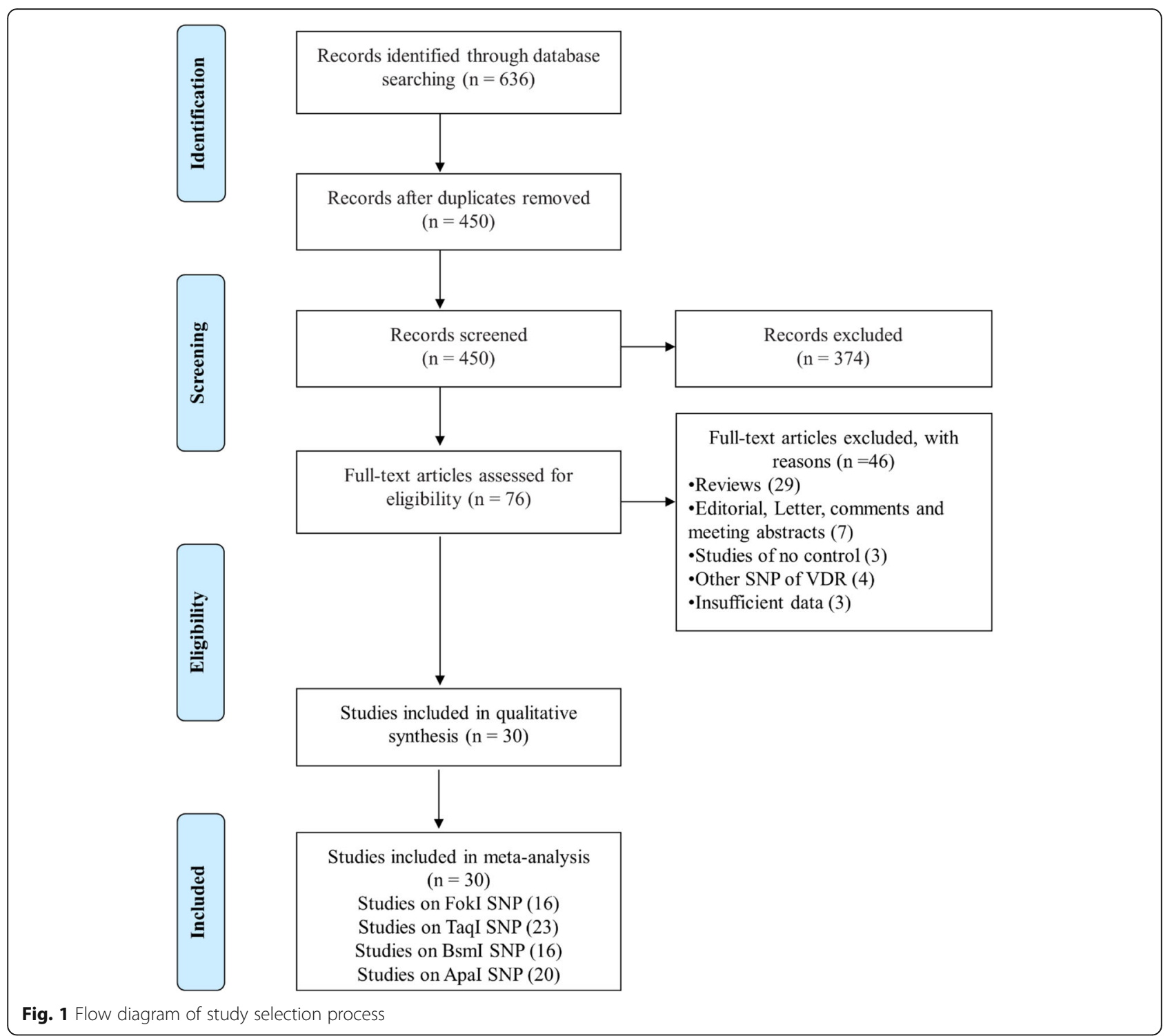

MS in European and Asian populations. Since there was only one study for American, and two studies for Australian populations, these studies were excluded from the subgroup analysis. The results of pooled ORs, heterogeneity tests and publication bias tests for different analysis models are shown in Table 3 (Additional file 1: Figures S1 and S2).

\section{Meta-analysis for Taql (rs731236) polymorphism and MS}

There were 23 case-control studies with 3758 cases and 3992 controls concerning TaqI polymorphism and MS risk. Among them, 13 studies were conducted in European countries [21, 22, 24, 34, 36, 37, 39, 40, 45-49], 5 studies in Asian countries [18, 41, 42, 50, 51], 2 studies in each Australian [17, 44] and American [43, 52] countries, and one study in Tunisia [20]. The TaqI polymorphism was demonstrated to be associated with MS risk under heterozygote contrast $(\mathrm{OR}=1.27,95 \% \mathrm{CI}=1.01-1.59$, random effect) (Fig. 2), whilst no significant association was detected across other genotype models (Table 3). In addition, the pooled results of subgroup analysis decline presence of significant association under all defined genetic model (Additional file 1: Figures S3 and S4). Groups with less than three studies were removed from subgroup analysis. The results of pooled ORs, heterogeneity tests and publication bias tests for different analysis models are shown in Table 3.

Meta-analysis for Bsml (rs1544410) polymorphism and MS After searching databases, finally 16 case-control studies with 1793 cases and 1815 controls subjects included to examine association between BsmI polymorphism and MS risk. Among 16 studies, six studies were performed in Europe [21, 22, 36, 40, 48, 49], eight studies in Asia $[18,41,50,51,53-55]$, and only two studies in America 
Table 1 Characteristics of studies included in Meta-analysis of overall MS

\begin{tabular}{|c|c|c|c|c|c|c|c|c|}
\hline Study author & Year & Country & Ethnicity & Sex cases/controls & $\begin{array}{l}\text { Total cases/ } \\
\text { control }\end{array}$ & $\begin{array}{l}\text { Age case/control } \\
\text { (Mean) }\end{array}$ & $\begin{array}{l}\text { Genotyping } \\
\text { method }\end{array}$ & $\begin{array}{l}\text { Quality } \\
\text { score }\end{array}$ \\
\hline \multicolumn{9}{|l|}{ Fokl (rs2228570) } \\
\hline Partridge et al. & 2004 & UK & European & $M=N R F=N R$ & $406 / 234$ & $43.8 \pm 11.2 / 50.1$ & NR & 7 \\
\hline Tajouri et al. & 2005 & Australia & Australian & $M=N R F=N R$ & $98 / 93$ & NR & PCR-RFLP & 6 \\
\hline Smolders et al. & 2009 & Netherland & European & $M=62 / 142 F=150 / 147$ & $212 / 289$ & $46.7 \pm 11.9 / 34.9 \pm 14.3$ & PCR-RFLP & 8 \\
\hline Dickinson et al. & 2009 & Australia & Australian & $M=N R F=N R$ & $136 / 235$ & $43.5 \pm 9.3 / 43.6 \pm 9.2$ & Taq-Man & 7 \\
\hline Simon et al. & 2010 & USA & American & $M=N R F=N R$ & $100 / 100$ & NR & Taq-Man & 7 \\
\hline Garcia-Martin et al. & 2013 & Spain & European & $M=94 / 98 F=209 / 212$ & $303 / 310$ & $43.9 \pm 11.4 / 43.4 \pm 11.7$ & Taq-Man & 8 \\
\hline Al-Temaimi et al. & 2015 & Kuwait & Asian & $M=17 / 19 F=33 / 31$ & $50 / 50$ & $33.4 \pm 9.6 / 28.6 \pm 7.9$ & Taq-Man & 7 \\
\hline Narooie-Nejad et al. & 2015 & Iran & Asian & $M=25 / 28 F=88 / 94$ & $113 / 122$ & $32.4 \pm 8.9 / 30.8 \pm 10.2$ & PCR-RFLP & 7 \\
\hline Cierny et al. & 2015 & Slovakia & European & $M=66 / 74 F=204 / 229$ & $270 / 303$ & $41.3 \pm 10.8 / 38.7 \pm 13.6$ & PCR-RFLP & 8 \\
\hline Luisa Agnello et al. & 2016 & Italy & European & $M=24 / 30 F=80 / 42$ & $104 / 75$ & $39.6 \pm 10.3 / 45.2 \pm 9.36$ & PCR-RFLP & 6 \\
\hline Abdollahzadeh et al. & 2016 & Iran & Asian & $M=40 / 38 F=120 / 112$ & $160 / 150$ & $35.9 \pm 3 / 36.8 \pm 1.8$ & PCR-RFLP & 7 \\
\hline Yucel et al. & 2017 & Turkey & European & $\mathrm{M}=\mathrm{NRF}=\mathrm{NR}$ & $29 / 114$ & $33.7 \pm 10.7 / 33.1 \pm 8.5$ & Taq-Man & 6 \\
\hline Bettencourt et al. & 2017 & Portugal & European & $M=185 / 198 F=348 / 248$ & $533 / 446$ & $30.2 \pm 9.3 / \mathrm{NR}$ & Taq-Man & 8 \\
\hline Kamisli et al. & 2018 & Turkey & European & $M=46 / 58 F=121 / 88$ & $167 / 146$ & $39.96 \pm 9.4 / 33.81 \pm 7.1$ & Taq-Man & 7 \\
\hline Sadeghi et al. & 2018 & Iran & Asian & $M=17 / 11 F=63 / 39$ & $80 / 50$ & $18-60 / 18-60$ & PCR-RFLP & 6 \\
\hline Křenek et al. & 2018 & Czech Republic & European & $M=80 / 49 F=216 / 86$ & 296/135 & $34-37$ / NR & PCR-RFLP & 7 \\
\hline \multicolumn{9}{|l|}{ Taql (rs731236) } \\
\hline Partridge et al. & 2004 & UK & European & $M=N R F=N R$ & $402 / 231$ & $43.8 \pm 11.2 / 50.1$ & NR & 7 \\
\hline Tajouri et al. & 2005 & Australia & Australian & $M=N R F=N R$ & $104 / 186$ & NR & PCR-RFLP & 6 \\
\hline Smolders et al. & 2009 & Netherland & European & $M=62 / 142 F=150 / 147$ & $212 / 289$ & $46.7 \pm 11.9 / 34.9 \pm 14.3$ & PCR-RFLP & 8 \\
\hline Dickinson et al. & 2009 & Australia & Australian & $\mathrm{M}=\mathrm{NR} F=\mathrm{NR}$ & $136 / 235$ & $43.5 \pm 9.3 / 43.6 \pm 9.2$ & Taq-Man & 7 \\
\hline Simon et al. & 2010 & USA & American & $\mathrm{M}=\mathrm{NR} F=\mathrm{NR}$ & $100 / 100$ & NR & Taq-Man & 7 \\
\hline Sioka et al. & 2011 & Greece & European & $M=23 / 23 F=46 / 58$ & $69 / 81$ & $39 \pm 10.5 / 38.7 \pm 10.7$ & Taq-Man & 7 \\
\hline Agliardi et al. & 2011 & Italy & European & $\mathrm{M}=\mathrm{NR} F=\mathrm{NR}$ & $641 / 558$ & $27.8 \pm 9.2 / 29.4 \pm 6.5$ & Taq-Man & 8 \\
\hline Irizar et al. & 2012 & Spain & European & $M=N R F=N R$ & $136 / 337$ & $44.14 \pm 13.02 / 50.17 \pm 13.26$ & PCR-SSP & 7 \\
\hline Garcia-Martin et al. & 2013 & Spain & European & $M=94 / 98 F=209 / 212$ & $303 / 310$ & $43.9 \pm 11.4 / 43.4 \pm 11.7$ & Taq-Man & 8 \\
\hline Selma et al. & 2015 & Tunisia & African & $M=22 / 47 F=38 / 67$ & $60 / 114$ & $35.8 \pm 6.3 / 37 \pm 9.3$ & PCR-RFLP & 6 \\
\hline Narooie-Nejad et al. & 2015 & Iran & Asian & $M=25 / 28 F=88 / 94$ & $113 / 122$ & $32.4 \pm 8.9 / 30.8 \pm 10.2$ & PCR-RFLP & 7 \\
\hline Al-Temaimi et al. & 2015 & Kuwait & Asian & $M=17 / 19 F=33 / 31$ & $50 / 50$ & $33.4 \pm 9.6 / 28.6 \pm 7.9$ & Taq-Man & 7 \\
\hline Yamout et al. & 2016 & Lebanon & Asian & $\mathrm{M}=\mathrm{NR} F=\mathrm{NR}$ & $50 / 99$ & $32.3 \pm 12.7 / 35.2 \pm 13$ & PCR-RFLP & 6 \\
\hline Cierny et al. & 2016 & Slovakia & European & $M=66 / 74 F=204 / 229$ & $270 / 303$ & $41.3 \pm 10.8 / 38.7 \pm 13.6$ & PCR-RFLP & 8 \\
\hline Luisa Agnello et al. & 2016 & Italy & European & $M=24 / 30 F=80 / 42$ & $104 / 75$ & $39.6 \pm 10.3 / 45.2 \pm 9.36$ & PCR-RFLP & 6 \\
\hline Terzi et al. & 2016 & Turkey & European & $\mathrm{M}=\mathrm{NR} F=\mathrm{NR}$ & $87 / 99$ & $30.149 .66 / N R$ & PCR-RFLP & 6 \\
\hline Abdollahzadeh et al. & 2016 & Iran & Asian & $M=40 / 38 F=120 / 112$ & $160 / 150$ & $35.9 \pm 3 / 36.8 \pm 1.8$ & PCR-RFLP & 7 \\
\hline Yucel et al. & 2017 & Turkey & European & $\mathrm{M}=\mathrm{NR} F=\mathrm{NR}$ & $28 / 72$ & $33.7 \pm 10.7 / 33.1 \pm 8.5$ & Taq-Man & 6 \\
\hline Kamisli et al. & 2018 & Turkey & European & $M=46 / 58 F=121 / 88$ & $167 / 146$ & $39.96 \pm 9.4 / 33.81 \pm 7.1$ & Taq-Man & 7 \\
\hline Morales et al. & 2018 & Mexico & American & $M=39 / 57 F=81 / 123$ & $120 / 180$ & $33.89 \pm 10.03 / 32.79 \pm 10.17$ & Taq-Man & 6 \\
\hline Sadeghi et al. & 2018 & Iran & Asian & $M=17 / 11 F=63 / 39$ & $80 / 50$ & $18-60 / 18-60$ & PCR-RFLP & 6 \\
\hline Cakina et al.. & 2018 & Turkey & European & $M=19 / 22 F=51 / 48$ & $70 / 70$ & $44.4 \pm 11.2 / 38.2 \pm 9.5$ & PCR-RFLP & 7 \\
\hline Křenek et al. & 2018 & Czech Republic & European & $M=80 / 49 F=216 / 86$ & $296 / 135$ & $34-37$ / NR & PCR-RFLP & 7 \\
\hline \multicolumn{9}{|l|}{ Bsml (rs1544410) } \\
\hline Fukazawa et al. & 1999 & Japan & Asian & $M=21 / 33 F=56 / 62$ & $77 / 95$ & $34.9 \pm 12.9 / \mathrm{NR}$ & PCR-RFLP & 8 \\
\hline Qinli Sun et al. & 2004 & China & Asian & $M=N R F=N R$ & $63 / 95$ & NR & PCR-RFLP & 6 \\
\hline Bing Wu et al. & 2009 & China & Asian & $M=N R F=N R$ & $83 / 120$ & NR & PCR-RFLP & 7 \\
\hline
\end{tabular}


Table 1 Characteristics of studies included in Meta-analysis of overall MS (Continued)

\begin{tabular}{|c|c|c|c|c|c|c|c|c|}
\hline Study author & Year & Country & Ethnicity & Sex cases/controls & $\begin{array}{l}\text { Total cases/ } \\
\text { control }\end{array}$ & $\begin{array}{l}\text { Age case/control } \\
\text { (Mean) }\end{array}$ & $\begin{array}{l}\text { Genotyping } \\
\text { method }\end{array}$ & $\begin{array}{l}\text { Quality } \\
\text { score }\end{array}$ \\
\hline Simon et al. & 2010 & USA & American & $M=N R F=N R$ & $101 / 100$ & NR & Taq-Man & 7 \\
\hline Sioka et al. & 2011 & Greece & European & $M=23 / 23 F=46 / 58$ & $69 / 81$ & $39 \pm 10.5 / 38.7 \pm 10.7$ & Taq-Man & 7 \\
\hline Al-Temaimi et al. & 2015 & Kuwait & Asian & $M=17 / 19 F=33 / 31$ & $50 / 50$ & $33.4 \pm 9.6 / 28.6 \pm 7.9$ & Taq-Man & 7 \\
\hline Narooie-Nejad et al. & 2015 & Iran & Asian & $M=25 / 28 F=88 / 94$ & $113 / 122$ & $32.4 \pm 8.9 / 30.8 \pm 10.2$ & PCR-RFLP & 7 \\
\hline Abdollahzadeh et al. & 2016 & Iran & Asian & $M=40 / 38 F=120 / 112$ & $160 / 150$ & $35.9 \pm 3 / 36.8 \pm 1.8$ & PCR-RFLP & 7 \\
\hline Yamout et al. & 2016 & Lebanon & Asian & $M=N R F=N R$ & $50 / 99$ & $32.3 \pm 12.7 / 35.2 \pm 13$ & PCR-RFLP & 6 \\
\hline Cierny et al. & 2016 & Slovakia & European & $M=66 / 74 F=204 / 229$ & $270 / 303$ & $41.3 \pm 10.8 / 38.7 \pm 13.6$ & PCR-RFLP & 8 \\
\hline Luisa Agnello et al. & 2016 & Italy & European & $M=24 / 30 F=80 / 42$ & $104 / 75$ & $39.6 \pm 10.3 / 45.2 \pm 9.36$ & PCR-RFLP & 6 \\
\hline Terzi et al. & 2016 & Turkey & European & $M=N R F=N R$ & $87 / 100$ & $30.14 \pm 9.66 / \mathrm{NR}$ & PCR-RFLP & 6 \\
\hline Morales et al. & 2017 & Mexico & American & $M=39 / 57 F=81 / 123$ & $120 / 180$ & $33.89 \pm 10.03 / 32.79 \pm 10.17$ & Taq-Man & 6 \\
\hline Sadeghi et al. & 2018 & Iran & Asian & $M=17 / 11 F=63 / 39$ & $80 / 50$ & $18-60 / 18-60$ & PCR-RFLP & 6 \\
\hline Cakina et al.. & 2018 & Turkey & European & $M=19 / 22 F=51 / 48$ & $70 / 70$ & $44.4 \pm 11.2 / 38.2 \pm 9.5$ & PCR-RFLP & 7 \\
\hline Křenek et al. & 2018 & Czech Republic & European & $M=80 / 49 F=216 / 86$ & $296 / 135$ & $34-37$ / NR & PCR-RFLP & 7 \\
\hline \multicolumn{9}{|l|}{ Apal (rs7975232) } \\
\hline Niino et al. & 2000 & Japan & Asian & $M=21 / 33 F=56 / 62$ & $77 / 95$ & $36.2 \pm 11.2 / 34.4 \pm 10.2$ & PCR-RFLP & 7 \\
\hline Qinli Sun et al. & 2004 & China & Asian & $M=N R F=N R$ & $63 / 95$ & NR & PCR-RFLP & 6 \\
\hline Tajouri et al. & 2005 & Australia & Australian & $M=N R F=N R$ & $104 / 100$ & NR & PCR-RFLP & 6 \\
\hline Smolders et al. & 2009 & Netherland & European & $M=62 / 142 F=150 / 147$ & $212 / 289$ & $46.7 \pm 11.9 / 34.9 \pm 14.3$ & PCR-RFLP & 8 \\
\hline Bing Wu et al. & 2009 & China & Asian & $M=N R F=N R$ & $83 / 120$ & NR & PCR-RFLP & 7 \\
\hline Simon et al. & 2010 & USA & American & $M=N R F=N R$ & $100 / 100$ & NR & Taq-Man & 7 \\
\hline Irizar et al. & 2012 & Spain & European & $M=N R F=N R$ & $134 / 340$ & $44.14 \pm 13.02 / 50.17 \pm 13.26$ & PCR-SSP & 7 \\
\hline Narooie-Nejad et al. & 2015 & Iran & Asian & $M=25 / 28 F=88 / 94$ & $113 / 122$ & $32.4 \pm 8.9 / 30.8 \pm 10.2$ & PCR-RFLP & 7 \\
\hline Al-Temaimi et al. & 2015 & Kuwait & Asian & $M=17 / 19 F=33 / 31$ & $50 / 50$ & $33.4 \pm 9.6 / 28.6 \pm 7.9$ & Taq-Man & 7 \\
\hline Selma et al. & 2015 & Tunisia & African & $M=22 / 47 F=38 / 67$ & $60 / 114$ & $35.8 \pm 6.3 / 37 \pm 9.3$ & PCR-RFLP & 6 \\
\hline Yamout et al. & 2016 & Lebanon & Asian & $\mathrm{M}=\mathrm{NR} F=\mathrm{NR}$ & $50 / 134$ & $32.3 \pm 12.7 / 35.2 \pm 13$ & PCR-RFLP & 6 \\
\hline Luisa Agnello et al. & 2016 & Italy & European & $M=24 / 30 F=80 / 42$ & $104 / 75$ & $39.6 \pm 10.3 / 45.2 \pm 9.36$ & PCR-RFLP & 6 \\
\hline Abdollahzadeh et al. & 2016 & Iran & Asian & $M=40 / 38 F=120 / 112$ & $160 / 150$ & $35.9 \pm 3 / 36.8 \pm 1.8$ & PCR-RFLP & 7 \\
\hline Cierny et al. & 2016 & Slovakia & European & $M=66 / 74 F=204 / 229$ & $270 / 303$ & $41.3 \pm 10.8 / 38.7 \pm 13.6$ & PCR-RFLP & 8 \\
\hline Terzi et al. & 2016 & Turkey & European & $M=N R F=N R$ & $87 / 100$ & $30.149 .66 / N R$ & PCR-RFLP & 6 \\
\hline Yucel et al. & 2017 & Turkey & European & $M=N R F=N R$ & $26 / 81$ & $33.7 \pm 10.7 / 33.1 \pm 8.5$ & Taq-Man & 6 \\
\hline Kamisli et al. & 2018 & Turkey & European & $M=46 / 58 F=121 / 88$ & $167 / 146$ & $39.96 \pm 9.4 / 33.81 \pm 7.1$ & Taq-Man & 8 \\
\hline Sadeghi et al. & 2018 & Iran & Asian & $M=17 / 11 F=63 / 39$ & $80 / 50$ & $18-60 / 18-60$ & PCR-RFLP & 7 \\
\hline Cakina et al.. & 2018 & Turkey & European & $M=19 / 22 F=51 / 48$ & $70 / 70$ & $44.4 \pm 11.2 / 38.2 \pm 9.5$ & PCR-RFLP & 7 \\
\hline Křenek et al. & 2018 & Czech Republic & European & $M=80 / 49 F=216 / 86$ & $296 / 135$ & $34-37$ / NR & PCR-RFLP & 7 \\
\hline
\end{tabular}

$N R$ not reported, $M$ male, $F$ female, $M S$ Multiple Sclerosis

continent $[43,52]$. The pooled results demonstrate no significant association between BsmI polymorphism and MS risk under all genetic models, but subgroup analysis revealed that BsmI polymorphism across recessive model increased the risk of MS in Asian population $(\mathrm{OR}=1.78$, $95 \% \mathrm{CI}=1.01-2.93$, random effect) compared to European population $(\mathrm{OR}=0.84,95 \% \mathrm{CI}=0.66-1.06$, random effect) (Fig. 3). The results of pooled ORs, heterogeneity tests and publication bias tests for different analysis models are shown in Table 3 (Additional file 1: Figures S5 and S6).

\section{Meta-analysis for Apa1 (rs7975232) polymorphism and} MS

For quantitative synthesis of association between ApaI polymorphism and MS risk, 20 case-control studies with 2306 cases and 2669 controls were identified to be eligible. Overall, nine studies in Europe [21, 36, 37, 39, 40, 45, 46, 48, 49], eight studies in Asia [18, 41, 42, 50, 51, 54-56], and one study in Africa [20], America [43] and Australia [17] were identified. There was no evidence of association between ApaI polymorphism and MS risk in the pooled results. However, subgroup analysis detected significant 
Table 2 Distribution of genotype and allele among MS patients and controls

\begin{tabular}{|c|c|c|c|c|c|c|c|c|c|c|c|c|}
\hline \multirow[t]{2}{*}{ Study author } & \multicolumn{5}{|c|}{ MS cases } & \multicolumn{5}{|c|}{ Healthy control } & \multirow[t]{2}{*}{ P-HWE } & \multirow[t]{2}{*}{ MAF } \\
\hline & $\mathrm{FF}$ & $\mathrm{Ff}$ & $\mathrm{ff}$ & $\mathrm{F}$ & $f$ & $\mathrm{FF}$ & $\mathrm{Ff}$ & $\mathrm{Ff}$ & $\mathrm{F}$ & $f$ & & \\
\hline \multicolumn{13}{|l|}{ Fokl (rs2228570) } \\
\hline Partridge et al. & 155 & 196 & 55 & 506 & 306 & 83 & 105 & 46 & 271 & 197 & $0 / 22$ & $0 / 42$ \\
\hline Tajouri et al. & 47 & 40 & 11 & 134 & 62 & 34 & 48 & 11 & 116 & 70 & $0 / 33$ & $0 / 376$ \\
\hline Smolders et al. & 79 & 103 & 30 & 261 & 163 & 113 & 134 & 42 & 360 & 218 & $0 / 82$ & $0 / 377$ \\
\hline Dickinson et al. & 58 & 61 & 17 & 177 & 95 & 86 & 110 & 39 & 282 & 188 & $0 / 72$ & $0 / 4$ \\
\hline Simon et al. & 36 & 45 & 19 & 117 & 83 & 41 & 44 & 15 & 126 & 74 & $0 / 57$ & $0 / 37$ \\
\hline Garcia-Martin et al. & 130 & 141 & 32 & 401 & 205 & 144 & 124 & 42 & 412 & 208 & 0/07 & $0 / 335$ \\
\hline Al-Temaimi et al. & 33 & 14 & 3 & 80 & 20 & 33 & 16 & 1 & 82 & 18 & $0 / 55$ & $0 / 18$ \\
\hline Narooie-Nejad et al. & 73 & 32 & 8 & 178 & 48 & 93 & 29 & 0 & 215 & 29 & $0 / 13$ & $0 / 118$ \\
\hline Cierny et al. & 96 & 143 & 31 & 335 & 205 & 118 & 143 & 42 & 379 & 227 & 0/89 & $0 / 374$ \\
\hline Luisa Agnello et al. & 34 & 52 & 18 & 120 & 88 & 29 & 36 & 10 & 94 & 56 & $0 / 82$ & $0 / 373$ \\
\hline Abdollahzadeh et al. & 14 & 67 & 79 & 95 & 225 & 11 & 59 & 80 & 81 & 219 & $0 / 97$ & $0 / 73$ \\
\hline Yucel et al. & 22 & 6 & 1 & 50 & 8 & 72 & 34 & 8 & 178 & 50 & $0 / 16$ & $0 / 219$ \\
\hline Bettencourt et al. & 223 & 227 & 83 & 673 & 393 & 204 & 197 & 45 & 605 & 287 & 0/79 & $0 / 321$ \\
\hline Kamisli et al. & 75 & 77 & 15 & 227 & 107 & 94 & 46 & 6 & 234 & 58 & 0/92 & 0/198 \\
\hline Sadeghi et al. & 47 & 32 & 1 & 126 & 34 & 20 & 26 & 4 & 66 & 34 & $0 / 26$ & $0 / 34$ \\
\hline Křenek et al. & 102 & 145 & 49 & 349 & 243 & 37 & 74 & 24 & 148 & 122 & $0 / 21$ & $0 / 451$ \\
\hline \multirow[t]{2}{*}{ Study author } & \multicolumn{5}{|c|}{ MS cases } & \multicolumn{5}{|c|}{ Healthy control } & P-HWE & MAF \\
\hline & $\pi$ & $\mathrm{Tt}$ & $\mathrm{tt}$ & $\mathrm{T}$ & t & $\pi$ & $\mathrm{Tt}$ & $\mathrm{tt}$ & $\mathrm{T}$ & T & & \\
\hline \multicolumn{13}{|l|}{ Taql (rs731236) } \\
\hline Partridge et al. & 140 & 203 & 59 & 483 & 321 & 86 & 106 & 39 & 278 & 184 & $0 / 51$ & $0 / 398$ \\
\hline Tajouri et al. & 27 & 57 & 20 & 111 & 97 & 104 & 42 & 40 & 250 & 122 & $0 / 57$ & $0 / 327$ \\
\hline Smolders et al. & 83 & 96 & 33 & 262 & 162 & 112 & 138 & 39 & 362 & 216 & $0 / 53$ & $0 / 373$ \\
\hline Dickinson et al. & 52 & 68 & 16 & 172 & 100 & 86 & 108 & 41 & 280 & 190 & $0 / 48$ & $0 / 4$ \\
\hline Simon et al. & 40 & 50 & 10 & 130 & 70 & 36 & 48 & 16 & 120 & 80 & 1 & $0 / 4$ \\
\hline Sioka et al. & 30 & 30 & 9 & 90 & 48 & 33 & 36 & 12 & 102 & 60 & $0 / 67$ & $0 / 37$ \\
\hline Agliardi et al. & 219 & 308 & 114 & 746 & 536 & 220 & 249 & 89 & 689 & 427 & 0/19 & $0 / 375$ \\
\hline Irizar et al. & 55 & 70 & 11 & 180 & 92 & 145 & 157 & 35 & 447 & 227 & $0 / 43$ & $0 / 336$ \\
\hline Garcia-Martin et al. & 129 & 129 & 45 & 387 & 219 & 131 & 144 & 35 & 406 & 214 & $0 / 62$ & $0 / 345$ \\
\hline Selma et al. & 28 & 29 & 3 & 85 & 35 & 75 & 38 & 1 & 188 & 40 & $0 / 1$ & $0 / 175$ \\
\hline Narooie-Nejad et al. & 9 & 44 & 60 & 62 & 164 & 94 & 26 & 2 & 214 & 30 & 0/89 & $0 / 122$ \\
\hline Al-Temaimi et al. & 31 & 19 & 0 & 81 & 19 & 15 & 28 & 7 & 58 & 42 & $0 / 29$ & $0 / 42$ \\
\hline Yamout et al. & 19 & 23 & 8 & 61 & 39 & 32 & 48 & 19 & 112 & 86 & 0/89 & $0 / 434$ \\
\hline Cierny et al. & 94 & 138 & 38 & 326 & 214 & 125 & 123 & 55 & 373 & 233 & 0/01 & $0 / 384$ \\
\hline Luisa Agnello et al. & 35 & 48 & 21 & 118 & 90 & 23 & 40 & 12 & 86 & 64 & $0 / 43$ & $0 / 426$ \\
\hline Terzi et al. & 30 & 43 & 14 & 103 & 71 & 48 & 43 & 9 & 137 & 61 & $0 / 85$ & $0 / 308$ \\
\hline Abdollahzadeh et al. & 38 & 80 & 42 & 156 & 164 & 63 & 68 & 19 & 194 & 106 & 0/92 & $0 / 353$ \\
\hline Yucel et al. & 13 & 15 & 0 & 41 & 15 & 31 & 26 & 15 & 88 & 56 & 0/05 & $0 / 388$ \\
\hline Kamisli et al. & 71 & 77 & 19 & 219 & 115 & 59 & 65 & 22 & 183 & 109 & $0 / 55$ & $0 / 373$ \\
\hline Morales et al. & 65 & 46 & 9 & 176 & 64 & 122 & 41 & 17 & 285 & 75 & 0/005 & $0 / 208$ \\
\hline Sadeghi et al. & 38 & 41 & 1 & 117 & 43 & 14 & 34 & 2 & 62 & 38 & 0/02 & $0 / 38$ \\
\hline Cakina et al. & 20 & 41 & 9 & 81 & 59 & 20 & 45 & 5 & 85 & 55 & 0/001 & $0 / 392$ \\
\hline Křenek et al. & 118 & 151 & 27 & 387 & 205 & 58 & 66 & 11 & 182 & 88 & 0/19 & $0 / 325$ \\
\hline
\end{tabular}


Table 2 Distribution of genotype and allele among MS patients and controls (Continued)

\begin{tabular}{|c|c|c|c|c|c|c|c|c|c|c|c|c|}
\hline \multirow[t]{2}{*}{ Study author } & \multicolumn{5}{|c|}{ MS cases } & \multicolumn{5}{|c|}{ Healthy control } & \multirow[t]{2}{*}{ P-HWE } & \multirow[t]{2}{*}{ MAF } \\
\hline & $\overline{\mathrm{FF}}$ & $\mathrm{Ff}$ & $\mathrm{ff}$ & $\mathrm{F}$ & $f$ & $\mathrm{FF}$ & $\mathrm{Ff}$ & $\mathrm{Ff}$ & $\mathrm{F}$ & f & & \\
\hline \multirow[t]{2}{*}{ Study author } & \multicolumn{5}{|c|}{ MS cases } & \multicolumn{5}{|c|}{ Healthy control } & P-HWE & MAF \\
\hline & BB & $\mathrm{Bb}$ & $\mathrm{bb}$ & $B$ & $b$ & BB & $\mathrm{Bb}$ & $\mathrm{bb}$ & B & B & & \\
\hline \multicolumn{13}{|l|}{ Bsml (rs1544410) } \\
\hline Fukazawa et al. & 0 & 11 & 66 & 11 & 143 & 3 & 24 & 68 & 30 & 160 & $0 / 62$ & $0 / 842$ \\
\hline Qinli Sun et al. & 0 & 7 & 56 & 7 & 119 & 0 & 11 & 84 & 11 & 179 & $0 / 54$ & 0/942 \\
\hline Bing Wu et al. & 0 & 5 & 78 & 5 & 161 & 0 & 26 & 94 & 26 & 214 & 0/18 & 0/891 \\
\hline Simon et al. & 39 & 49 & 13 & 127 & 75 & 34 & 47 & 19 & 115 & 85 & 0/71 & $0 / 425$ \\
\hline Sioka et al. & 28 & 41 & 0 & 97 & 41 & 26 & 55 & 0 & 107 & 55 & 0/004 & $0 / 339$ \\
\hline Al-Temaimi et al. & 20 & 30 & 0 & 70 & 30 & 15 & 25 & 10 & 55 & 45 & $0 / 94$ & $0 / 45$ \\
\hline Narooie-Nejad et al. & 59 & 50 & 4 & 168 & 58 & 45 & 65 & 12 & 155 & 89 & 0/09 & $0 / 364$ \\
\hline Abdollahzadeh et al. & 46 & 79 & 35 & 171 & 149 & 70 & 65 & 15 & 205 & 95 & 0/98 & $0 / 316$ \\
\hline Yamout et al. & 10 & 21 & 19 & 41 & 59 & 16 & 53 & 30 & 85 & 113 & $0 / 35$ & $0 / 57$ \\
\hline Cierny et al. & 43 & 139 & 88 & 225 & 315 & 73 & 111 & 119 & 257 & 349 & 0/001 & $0 / 575$ \\
\hline Luisa Agnello et al. & 23 & 48 & 33 & 94 & 114 & 17 & 37 & 21 & 71 & 79 & 0/92 & $0 / 526$ \\
\hline Terzi et al. & 19 & 40 & 28 & 78 & 96 & 14 & 47 & 39 & 75 & 125 & 0/97 & $0 / 625$ \\
\hline Morales et al. & 60 & 38 & 22 & 158 & 82 & 110 & 60 & 10 & 280 & 80 & 0/63 & $0 / 222$ \\
\hline Sadeghi et al. & 12 & 51 & 17 & 75 & 85 & 16 & 29 & 5 & 61 & 39 & $0 / 12$ & $0 / 39$ \\
\hline Cakina et al.. & 14 & 36 & 20 & 64 & 76 & 11 & 37 & 22 & 59 & 81 & $0 / 48$ & $0 / 578$ \\
\hline Křenek et al. & 114 & 153 & 29 & 381 & 211 & 61 & 63 & 11 & 185 & 85 & $0 / 34$ & $0 / 314$ \\
\hline \multirow[t]{2}{*}{ Study author } & \multicolumn{5}{|c|}{ MS cases } & \multicolumn{5}{|c|}{ Healthy control } & P-HWE & MAF \\
\hline & AA & $\mathrm{Aa}$ & aa & A & $\mathrm{a}$ & $\mathrm{AA}$ & $\mathrm{Aa}$ & aа & A & A & & \\
\hline \multicolumn{13}{|l|}{ Apal (rs7975232) } \\
\hline Niino et al. & 21 & 23 & 33 & 65 & 89 & 9 & 41 & 45 & 59 & 131 & 0/93 & $0 / 689$ \\
\hline Qinli Sun & 9 & 17 & 37 & 35 & 91 & 15 & 29 & 51 & 59 & 131 & 0/005 & $0 / 689$ \\
\hline Tajouri et al. & 35 & 55 & 14 & 125 & 83 & 23 & 54 & 23 & 100 & 100 & $0 / 42$ & $0 / 5$ \\
\hline Smolders et al. & 58 & 99 & 55 & 215 & 209 & 80 & 150 & 59 & 310 & 268 & $0 / 45$ & $0 / 463$ \\
\hline Bing Wu et al. & 14 & 39 & 30 & 67 & 99 & 10 & 45 & 65 & 65 & 175 & $0 / 58$ & $0 / 729$ \\
\hline Simon et al. & 29 & 45 & 26 & 103 & 97 & 28 & 50 & 22 & 106 & 94 & 0/97 & $0 / 47$ \\
\hline Irizar et al. & 39 & 60 & 35 & 138 & 130 & 76 & 178 & 86 & 330 & 350 & $0 / 37$ & $0 / 514$ \\
\hline Narooie-Nejad et al. & 40 & 62 & 11 & 142 & 84 & 61 & 56 & 5 & 178 & 66 & 0/07 & $0 / 27$ \\
\hline Al-Temaimi et al. & 20 & 25 & 5 & 65 & 35 & 23 & 17 & 10 & 63 & 37 & 0/05 & $0 / 37$ \\
\hline Selma et al. & 14 & 36 & 10 & 64 & 56 & 40 & 58 & 16 & 138 & 90 & $0 / 48$ & $0 / 394$ \\
\hline Yamout et al. & 19 & 22 & 9 & 60 & 40 & 33 & 51 & 15 & 117 & 81 & $0 / 51$ & $0 / 503$ \\
\hline Luisa Agnello et al. & 31 & 58 & 15 & 120 & 88 & 26 & 41 & 8 & 93 & 57 & $0 / 16$ & $0 / 38$ \\
\hline Abdollahzadeh et al. & 18 & 67 & 75 & 103 & 217 & 4 & 43 & 103 & 51 & 249 & $0 / 84$ & $0 / 83$ \\
\hline Cierny et al. & 78 & 132 & 60 & 288 & 252 & 102 & 120 & 81 & 324 & 282 & 0/005 & $0 / 465$ \\
\hline Terzi et al. & 28 & 46 & 13 & 102 & 72 & 42 & 40 & 18 & 124 & 76 & $0 / 13$ & $0 / 38$ \\
\hline Yucel et al. & 8 & 13 & 5 & 29 & 23 & 28 & 37 & 16 & 93 & 69 & $0 / 55$ & $0 / 425$ \\
\hline Kamisli et al. & 62 & 76 & 29 & 200 & 134 & 54 & 67 & 25 & 175 & 117 & $0 / 59$ & $0 / 4$ \\
\hline Sadeghi et al. & 22 & 53 & 5 & 97 & 63 & 23 & 22 & 5 & 68 & 32 & 0/93 & $0 / 32$ \\
\hline Cakina et al.. & 28 & 27 & 15 & 83 & 57 & 20 & 21 & 29 & 61 & 79 & 0/001 & $0 / 564$ \\
\hline Křenek et al. & 27 & 183 & 86 & 237 & 355 & 31 & 78 & 26 & 140 & 130 & 0/07 & $0 / 481$ \\
\hline
\end{tabular}


Table 3 Main results of pooled ORs in meta-analysis of Vitamin D Receptor Gene Polymorphisms

\begin{tabular}{|c|c|c|c|c|c|c|c|c|c|c|}
\hline \multirow[t]{2}{*}{ Subgroup } & \multirow[b]{2}{*}{ Genetic model } & \multirow{2}{*}{$\begin{array}{l}\text { Sample size } \\
\text { Case/Control }\end{array}$} & \multicolumn{2}{|c|}{$\begin{array}{l}\text { Test of } \\
\text { association }\end{array}$} & \multicolumn{2}{|c|}{$\begin{array}{l}\text { Test of } \\
\text { heterogeneity }\end{array}$} & \multicolumn{2}{|c|}{$\begin{array}{l}\text { Test of publication bias } \\
\text { (Begg's test) }\end{array}$} & \multicolumn{2}{|c|}{$\begin{array}{l}\text { Test of publication bias } \\
\text { (Egger's test) }\end{array}$} \\
\hline & & & $\overline{\mathrm{OR}}$ & $95 \% \mathrm{Cl}$ & $\overline{P^{2}(\%)}$ & $P$ & $\bar{Z}$ & $P$ & $\overline{\mathrm{T}}$ & $P$ \\
\hline \multicolumn{11}{|c|}{ Fokl (rs2228570) } \\
\hline \multirow[t]{5}{*}{ Overall } & Dominant model & 3057 / 2852 & 1.06 & $0.94-1.19$ & 45.7 & 0.02 & -1.44 & 0.15 & -1.09 & 0.29 \\
\hline & Recessive model & 3057 / 2852 & 0.96 & $0.81-1.13$ & 23.8 & 0.14 & 0.78 & 0.45 & 0.13 & 0.90 \\
\hline & Allelic model & 3057 / 2852 & 1.08 & $0.93-1.26$ & 66.6 & $\leq 0.001$ & 0.63 & 0.52 & 0.46 & 0.65 \\
\hline & ff vs. FF & 3057 / 2852 & 0.96 & $0.80-1.16$ & 48.4 & 0.01 & 0.05 & 0.96 & -0.63 & 0.54 \\
\hline & $\mathrm{Ff}$ vs.FF & 3057 / 2852 & 1.06 & $0.93-1.19$ & 26.4 & 0.16 & -1.44 & 0.15 & -1.33 & 0.20 \\
\hline \multirow[t]{5}{*}{ European } & Dominant model & 2480 / 2202 & 1.10 & $0.97-1.26$ & 41.7 & 0.08 & -1.16 & 0.24 & -0.62 & 0.55 \\
\hline & Recessive model & 2480 / 2202 & 0.96 & $0.80-1.15$ & 38.1 & 0.10 & 1.16 & 0.24 & 0.20 & 0.84 \\
\hline & Allelic model & 2480 / 2202 & 1.04 & $0.90-1.20$ & 56.5 & 0.01 & 0.27 & 0.78 & -0.32 & 0.75 \\
\hline & ff vs. FF & 2480 / 2202 & 1.00 & $0.75-1.33$ & 42.8 & 0.07 & 0.27 & 0.78 & -0.17 & 0.86 \\
\hline & Ff vs.FF & 2480 / 2202 & 1.11 & $0.97-1.28$ & 24.9 & 0.21 & 0.27 & 0.78 & 0.24 & 0.81 \\
\hline \multirow[t]{5}{*}{ Asian } & Dominant model & $243 / 222$ & 1.05 & $0.68-1.61$ & 70.9 & 0.03 & -0.52 & 0.60 & -0.86 & 0.54 \\
\hline & Recessive model & $243 / 222$ & 1.27 & $0.21-7.61$ & 57.9 & 0.12 & -1 & 0.31 & * & * \\
\hline & Allelic model & $243 / 222$ & 1.06 & $0.46-2.45$ & 80.9 & $\leq 0.001$ & -0.52 & 0.60 & -0.36 & 0.77 \\
\hline & ff vs. FF & $243 / 222$ & 0.51 & $0.02-14.1$ & 84 & 0.01 & 1 & 0.31 & * & * \\
\hline & Ff vs. FF & $243 / 222$ & 0.93 & $0.60-1.45$ & 43.5 & 0.17 & -0.52 & 0.60 & -1.02 & 0.42 \\
\hline \multicolumn{11}{|c|}{ Taql (rs731236) } \\
\hline \multirow[t]{5}{*}{ Overall } & Dominant model & $3758 / 3992$ & 1.26 & $0.99-1.60$ & 80.5 & $\leq 0.001$ & -0.13 & 0.89 & 0.38 & 0.71 \\
\hline & Recessive model & $3758 / 3992$ & 0.19 & $0.91-1.57$ & 63 & $\leq 0.001$ & 1.75 & 0.08 & 1.46 & 0.16 \\
\hline & Allelic model & $3758 / 3992$ & 1.16 & $0.94-1.42$ & 87.2 & $\leq 0.001$ & -0.87 & 0.38 & 0.24 & 0.81 \\
\hline & tt vs. TT & $3758 / 3992$ & 1.26 & $0.93-1.71$ & 65.9 & $\leq 0.001$ & 0.54 & 0.58 & 0.98 & 0.34 \\
\hline & Tt vs.TT & $3758 / 3992$ & 1.27 & $1.01-1.59$ & 74.5 & $\leq 0.001$ & -0.50 & 0.61 & 0.43 & 0.67 \\
\hline \multirow[t]{5}{*}{ European } & Dominant model & $2785 / 2706$ & 1.11 & $0.99-1.25$ & 0 & 0.90 & -0.80 & 0.42 & 0.08 & 0.94 \\
\hline & Recessive model & $2785 / 2706$ & 1.04 & $0.88-1.23$ & 0 & 0.63 & 1.17 & 0.24 & 1.14 & 0.27 \\
\hline & Allelic model & $2785 / 2706$ & 1.06 & $0.97-1.15$ & 0 & 0.76 & -1.55 & 0.12 & 0.05 & 0.96 \\
\hline & tt vs. TT & $2785 / 2706$ & 1.11 & $0.92-1.33$ & 0 & 0.82 & 0.63 & 0.52 & 0.98 & 0.34 \\
\hline & Tt vs.TT & $2785 / 2706$ & 1.12 & $0.99-1.27$ & 0 & 0.79 & -0.72 & 0.47 & -0.08 & 0.94 \\
\hline \multirow[t]{5}{*}{ Asian } & Dominant model & 453 / 471 & 1.53 & $0.34-6.95$ & 95 & $\leq 0.001$ & -0.98 & 0.32 & -0.24 & 0.82 \\
\hline & Recessive model & $453 / 471$ & 2.98 & $0.55-16.2$ & 84.4 & $\leq 0.001$ & 0 & 1 & 0.22 & 0.84 \\
\hline & Allelic model & 453 / 471 & 1.43 & $0.40-5.13$ & 96.9 & $\leq 0.001$ & -1.96 & 0.05 & -0.59 & 0.59 \\
\hline & tt vs. TT & $453 / 471$ & 4.13 & $0.41-41.8$ & 90.5 & $\leq 0.001$ & 0 & 1 & 0.18 & 0.87 \\
\hline & Tt vs.TT & $453 / 471$ & 1.31 & $0.38-4.54$ & 91.7 & $\leq 0.001$ & -0.98 & 0.32 & -0.25 & 0.81 \\
\hline \multicolumn{11}{|c|}{ Bsml(rs1544410) } \\
\hline & Dominant model & $1793 / 1815$ & 0.84 & $0.48-1.49$ & 91.3 & $\leq 0.001$ & -0.24 & 0.80 & 1.81 & 0.09 \\
\hline & Recessive model & $1793 / 1815$ & 1.30 & $0.92-1.85$ & 62.9 & $\leq 0.001$ & 0.93 & 0.35 & 1.67 & 0.12 \\
\hline & Allelic model & $1793 / 1815$ & 1.10 & $0.89-1.37$ & 69.7 & $\leq 0.001$ & 0.18 & 0.85 & 0.23 & 0.82 \\
\hline & bb vs. BB & $1793 / 1815$ & 1.24 & $0.78-1.99$ & 64.9 & $\leq 0.001$ & -1.95 & 0.05 & -0.55 & 0.59 \\
\hline & $\mathrm{Bb}$ vs.BB & $1793 / 1815$ & 1.15 & $0.96-1.37$ & 49.6 & 0.02 & -1.46 & 0.14 & -1.65 & 0.12 \\
\hline \multirow[t]{4}{*}{ European } & Dominant model & $896 / 764$ & 0.62 & $0.25-1.55$ & 93.3 & $\leq 0.001$ & -0.19 & 0.85 & 1.50 & 0.2 \\
\hline & Recessive model & $896 / 764$ & 0.84 & $0.65-1.09$ & 0 & 0.66 & 1.47 & 0.14 & 1.84 & 0.16 \\
\hline & Allelic model & $896 / 764$ & 0.99 & $0.85-1.16$ & 0 & 0.51 & -0.94 & 0.34 & -1.45 & 0.22 \\
\hline & bb vs. BB & $896 / 764$ & 1.07 & $0.76-1.50$ & 0 & 0.46 & -1.96 & 0.05 & -1.29 & 0.28 \\
\hline
\end{tabular}


Table 3 Main results of pooled ORs in meta-analysis of Vitamin D Receptor Gene Polymorphisms (Continued)

\begin{tabular}{|c|c|c|c|c|c|c|c|c|c|c|}
\hline \multirow[t]{2}{*}{ Subgroup } & \multirow[b]{2}{*}{ Genetic model } & \multirow{2}{*}{$\begin{array}{l}\text { Sample size } \\
\text { Case/Control }\end{array}$} & \multicolumn{2}{|c|}{$\begin{array}{l}\text { Test of } \\
\text { association }\end{array}$} & \multicolumn{2}{|c|}{$\begin{array}{l}\text { Test of } \\
\text { heterogeneity }\end{array}$} & \multicolumn{2}{|c|}{$\begin{array}{l}\text { Test of publication bias } \\
\text { (Begg's test) }\end{array}$} & \multicolumn{2}{|c|}{$\begin{array}{l}\text { Test of publication bias } \\
\text { (Egger's test) }\end{array}$} \\
\hline & & & $\overline{\mathrm{OR}}$ & $95 \% \mathrm{Cl}$ & $\overline{1^{2}(\%)}$ & $P$ & $\bar{Z}$ & $P$ & $\overline{\mathrm{T}}$ & $P$ \\
\hline & $\mathrm{Bb}$ vs. $\mathrm{BB}$ & $896 / 764$ & 1.08 & $0.72-1.62$ & 54.1 & 0.05 & -0.94 & 0.34 & -2.34 & 0.07 \\
\hline \multirow[t]{5}{*}{ Asian } & Dominant model & $676 / 771$ & 1.09 & $0.54-2.22$ & 78.5 & $\leq 0.001$ & 0 & 1 & -0.30 & 0.78 \\
\hline & Recessive model & $676 / 771$ & 1.78 & $1.08-2.93$ & 44.2 & 0.09 & -1.35 & 0.17 & -1.01 & 0.35 \\
\hline & Allelic model & $676 / 771$ & 1.28 & $0.81-2.02$ & 79 & $\leq 0.001$ & -0.49 & 0.69 & 0.26 & 0.80 \\
\hline & bb vs. BB & $676 / 771$ & 1.50 & $0.46-4.88$ & 76.3 & $\leq 0.001$ & -1.36 & 0.17 & -0.97 & 0.43 \\
\hline & $\mathrm{Bb}$ vs.BB & $676 / 771$ & 1.08 & 0.59-1.96 & 66.9 & 0.01 & -0.49 & 0.62 & -0.12 & 0.91 \\
\hline \multicolumn{11}{|c|}{ Apal (rs7975232) } \\
\hline & Dominant model & $2306 / 2669$ & 1.04 & $0.82-1.31$ & 58 & $\leq 0.001$ & -1.23 & 0.21 & -1.14 & 0.26 \\
\hline & Recessive model & $2306 / 2669$ & 0.83 & $0.66-1.05$ & 51 & $\leq 0.001$ & -0.58 & 0.55 & -0.57 & 0.57 \\
\hline & Allelic model & $2306 / 2669$ & 0.94 & $0.80-1.10$ & 68.2 & $\leq 0.001$ & -0.55 & 0.58 & -1.02 & 0.32 \\
\hline & aa vs. AA & $2306 / 2669$ & 0.85 & $0.63-1.16$ & 55.1 & $\leq 0.001$ & -0.78 & 0.43 & -1.26 & 0.22 \\
\hline & Aa vs. AA & $2306 / 2669$ & 1.20 & $0.88-1.64$ & 72.2 & $\leq 0.001$ & -0.97 & 0.33 & -0.64 & 0.53 \\
\hline \multirow[t]{5}{*}{ European } & Dominant model & $1366 / 1539$ & 1.13 & $0.87-1.47$ & 49.8 & 0.04 & 0 & 1 & 0.21 & 0.84 \\
\hline & Recessive model & $1366 / 1539$ & 1.01 & $0.78-1.33$ & 38.7 & 0.11 & -0.42 & 0.67 & -0.49 & 0.64 \\
\hline & Allelic model & $1366 / 1539$ & 1.05 & $0.88-1.24$ & 53.6 & 0.02 & 0 & 1 & -0.56 & 0.59 \\
\hline & aa vs. AA & 1366 / 1539 & 1.11 & $0.76-1.63$ & 56.9 & 0.01 & 0.83 & 0.40 & 0.06 & 0.95 \\
\hline & Aa vs. AA & $1366 / 1539$ & 1.41 & $0.86-2.31$ & 81.4 & $\leq 0.001$ & 0.83 & 0.40 & 0.52 & 0.61 \\
\hline \multirow[t]{5}{*}{ Asian } & Dominant model & $676 / 816$ & 0.87 & $0.49-1.53$ & 70.9 & $\leq 0.001$ & -1.73 & 0.08 & -2.67 & 0.03 \\
\hline & Recessive model & $676 / 816$ & 0.61 & $0.42-0.89$ & 40.4 & 0.11 & 0.99 & 0.32 & 0.64 & 0.54 \\
\hline & Allelic model & $676 / 816$ & 0.81 & $0.57-1.15$ & 76.1 & $\leq 0.001$ & 1.37 & 0.17 & 0.69 & 0.51 \\
\hline & aa vs. AA & $676 / 816$ & 0.52 & $0.32-0.86$ & 28 & 0.20 & 0.25 & 0.80 & 0.37 & 0.72 \\
\hline & Aa vs. AA & $676 / 816$ & 1.03 & $0.59-1.79$ & 64.5 & $\leq 0.001$ & -2.23 & 0.02 & -2.17 & 0.07 \\
\hline
\end{tabular}

The significant values are presented with boldface

association between presence of ApaI SNP and risk of MS under recessive model $(\mathrm{OR}=0.61,95 \% \mathrm{CI}=0.42-0.89$, random effect) and homozygote model $(\mathrm{OR}=0.52$, $95 \% \mathrm{CI}=0.32-0.86$, random effect) in Asian population in comparison with European population $(\mathrm{OR}=1.01$, $95 \% \mathrm{CI}=0.78-1.33$, recessive model $)$ and $(\mathrm{OR}=1.11$, $95 \% \mathrm{CI}=0.76-1.63$, homozygote model) (Fig. 3). The results of pooled ORs, heterogeneity tests, and publication bias tests for different analysis models are shown in Table 3 (Additional file 1: Figures S7 and S8).

\section{Evaluation of heterogeneity}

Significant heterogeneity existed for FokI, TaqI, BsmI, and ApaI polymorphism in all of the genetic models. Furthermore, in subgroup analysis, there was a significant heterogeneity for studies carried out in Asian and European countries (Table 3).

\section{Publication bias and Sensitivity analysis}

Publication bias was estimated using funnel plot, Begg's and Egger's tests. No evidence of publication bias was seen for all four SNP and subgroup analysis under all genetic models. Additionally, the shape of the funnel plot appeared to be symmetrical, indicating that there was no significant publication bias (Fig. 4).The impact of individual study on pooled OR was estimated by sequential omission of each studies which results showed that no individual study significantly affected the pooled ORs under any genetic models of the VDR SNPs (Fig. 5).

\section{Discussion}

$V D R$ gene, as a pleiotropic gene, has been shown to be associated with several diseases. In the previous studies, the relationship between VDR gene single nucleotide polymorphisms and autoimmune disorders was evaluated in several meta-analyses. The study of Feng et al. [57]. described that TaqI or BsmI polymorphism in VDR gene was significantly connected with autoimmune thyroid diseases. Mao et al. [58]. represented that the BsmI B allele may act as a risk factor for onset of systemic lupus erythematous (SLE) among Asians and overall populations and also the FokI FF genotype act as a potential risk factor for SLE predisposition in Asians. Furthermore, Tizaoui 


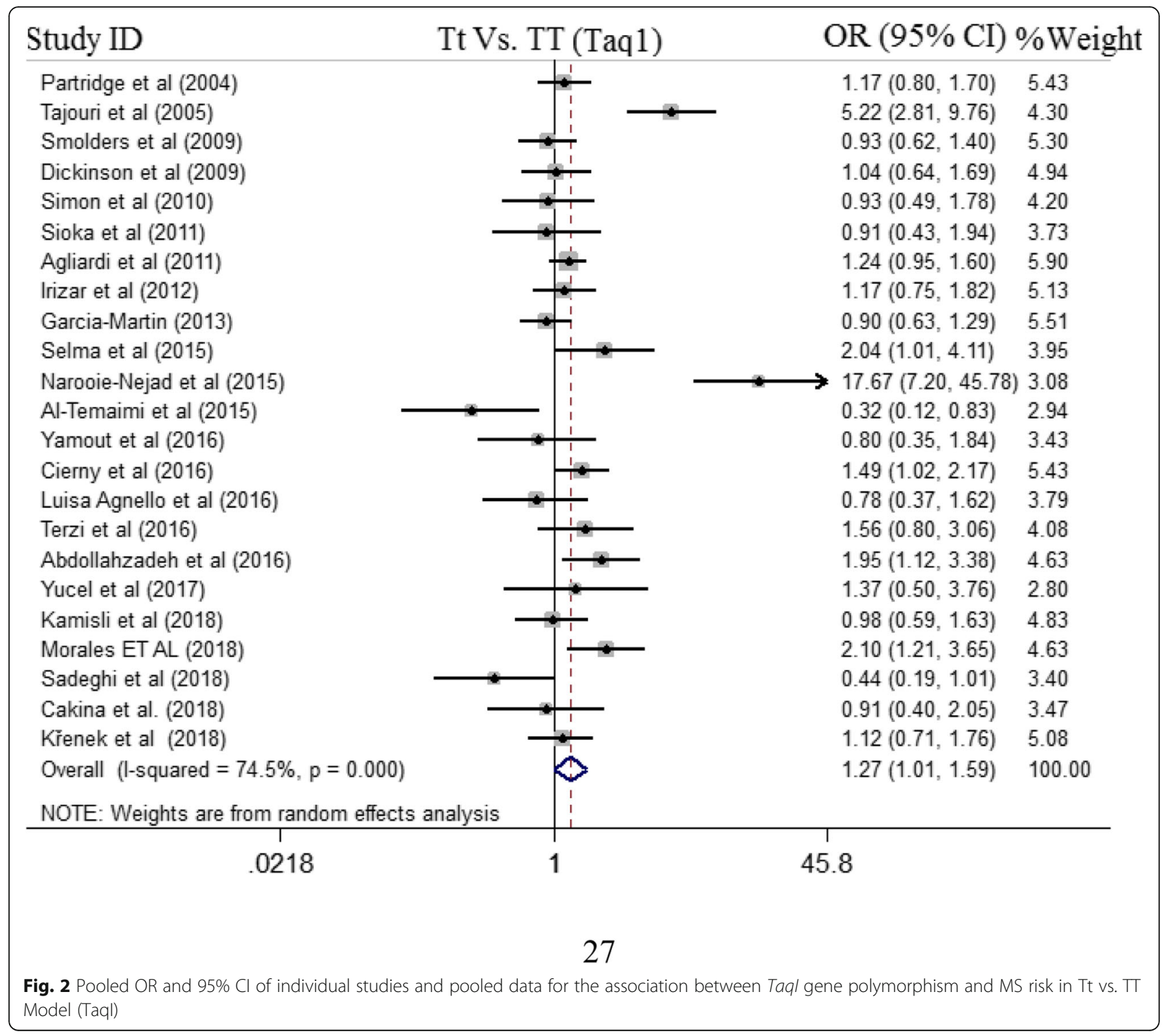

et al. [59] showed that the $V D R$ gene TaqI and FokI polymorphisms may increase the risk of Rheumatoid arthritis (RA) in European populations. And finally, Wang et al. [60] reported that the ApaI and BsmI polymorphisms were related with elevated susceptibility to type 1 diabetes (T1D) in Asian populations. Collectively, it could be assumed that $V D R$ gene polymorphisms act as a potential risk factor in development or progression of autoimmune disorders.

Although four meta-analyses have been conducted in the past 10 years to evaluate the relationship between the VDR gene polymorphisms and MS, these findings were inconclusive due to the variations of the literature and selected databases. Hence, for resolving these inconsistencies, and to decrease the heterogeneity and the probability of random errors, we set out an updated meta-analysis. In this study, 30 studies met the inclusion criteria and were included for quantitative synthesis. No evidence of publication bias was observed for all four SNP in subgroup analysis and overall populations under five genetic models. Regarding the essential role of genetic factors in the pathogenesis of MS, we categorized our results according to ethnicity. Our meta-analysis revealed that BsmI, ApaI, and TaqI polymorphisms may play a significant role in the pathogenesis of MS in overall and Asian population. The results of this study indicated that TaqI polymorphism was associated with MS susceptibility under heterozygote contrast in overall population.

Subgroup analysis based on continent rejected any association between $V D R$ gene polymorphisms and the MS risk in European population. However, a significant association between BsmI and ApaI polymorphisms and MS susceptibility was detected in Asian population. 

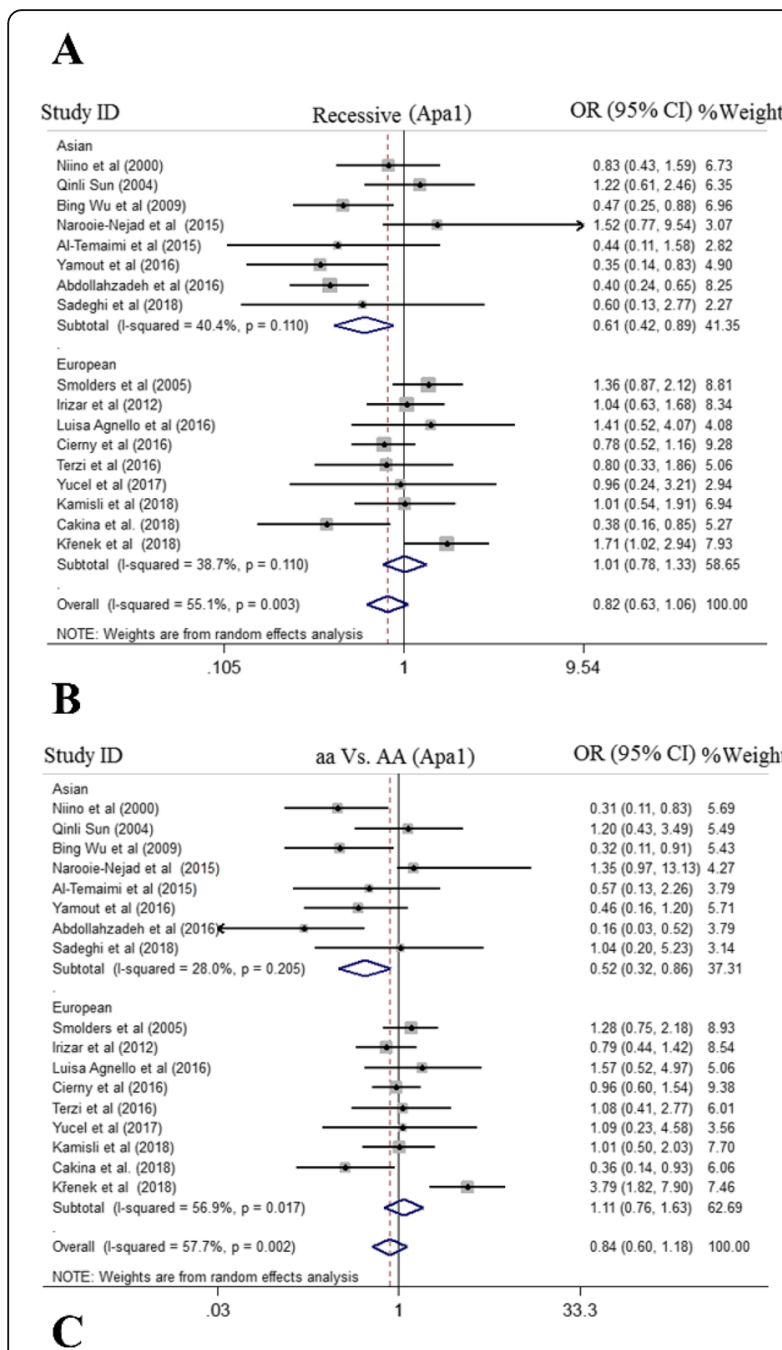

Study ID $\quad$ Recessive (Bsm1) OR $(95 \% \mathrm{CI}) \%$ Weight

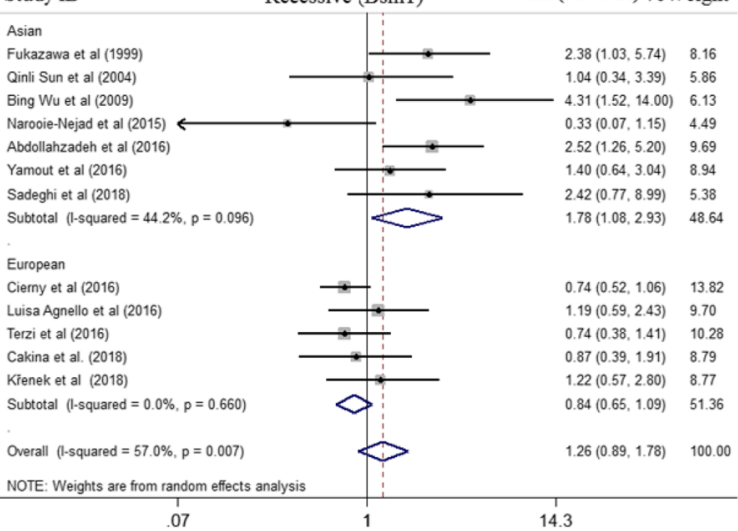

Fig. 3 Pooled odds ratio (OR)) and 95\% confidence interval of individual studies and pooled data for the association between Bsml, Apal gene polymorphism and MS risk in different ethnicity subgroups and overall populations for A; Recessive Model (Apal), B; aa vs. AA Model (Apal), and C; Recessive Model (Bsm)
BsmI polymorphism was associated with $64 \%$ increased risk of MS under recessive model in Asian populations. On the other hand, ApaI polymorphism was associated with 37.5 and $34.5 \%$ decreased risk of MS under recessive model and aa vs. AA model in Asian populations, respectively. The possible reason of the conflicting results among Asian, European, and overall population could be related to environmental factors the individuals exposed to and different genetic backgrounds, which may have disproportionate effects on MS risk.

These findings are inconsistent with the results of the Huang et al. [23] and Garcia-Martin et al. [24] studies. In the study of Huang et al.., 11 case-control studies with 2599 cases and 2816 controls were included for assessing the association between VDR gene polymorphisms and the MS susceptibility, but no significant association was found. Another study by Garcia-Martin et al. that analyzed ten studies with 2944 MS patients and 3166 healthy subjects, reported that TaqI and FokI polymorphisms were not associated with the MS risk. In accordance with our study, the study of Zhang et al. [25]. and Tizaoui et al. [26]. showed a significant association between $V D R$ gene polymorphisms and the MS susceptibility. However, there are some obvious differences in the findings of these studies in comparison with our study. Meta-analysis of Tizaoui et al.. reported an association of the FokI FF and ApaI AA genotypes with an elevated susceptibility of MS in a total of 3300 MS patients and 3194 healthy subjects from 13 case-control studies. In contrast, our analysis, consisting of 20 casecontrol studies, showed that ApaI polymorphism was associated with decreased risk of MS in Asian populations. In addition, the study of Zhang et al. reported that the A allele was related with the onset of disease in Asian populations. Nevertheless, the sensitivity analysis, by removing the studies not in HWE, rejected any association between the A allele and risk of MS, which was dissimilar to the results of the non-sensitivity analysis. Moreover, they failed to find any association between TaqI, BsmI, and ApaI polymorphisms and MS susceptibility in overall populations, Asians, and Caucasians. The main reasons that $V D R$ gene polymorphism plays a diverse function across different studies or in different ethnic populations may be due to the following hints. Firstly, in many cases, controls in included studies deviated from HWE. Secondly, the differences in the ethnic contextual characteristics of the patients may be an important factor for these variations. Thirdly, VDR SNPs were suggested to be related with the basal levels of 1 , 25(OH)2D3 and vitamin D structure and function [61], which in turn could influence MS predisposition. Finally, MS is regarded to be a polygenic disorder, and therefore it is expected that various gene loci are interacted in the pathogenesis of MS. 

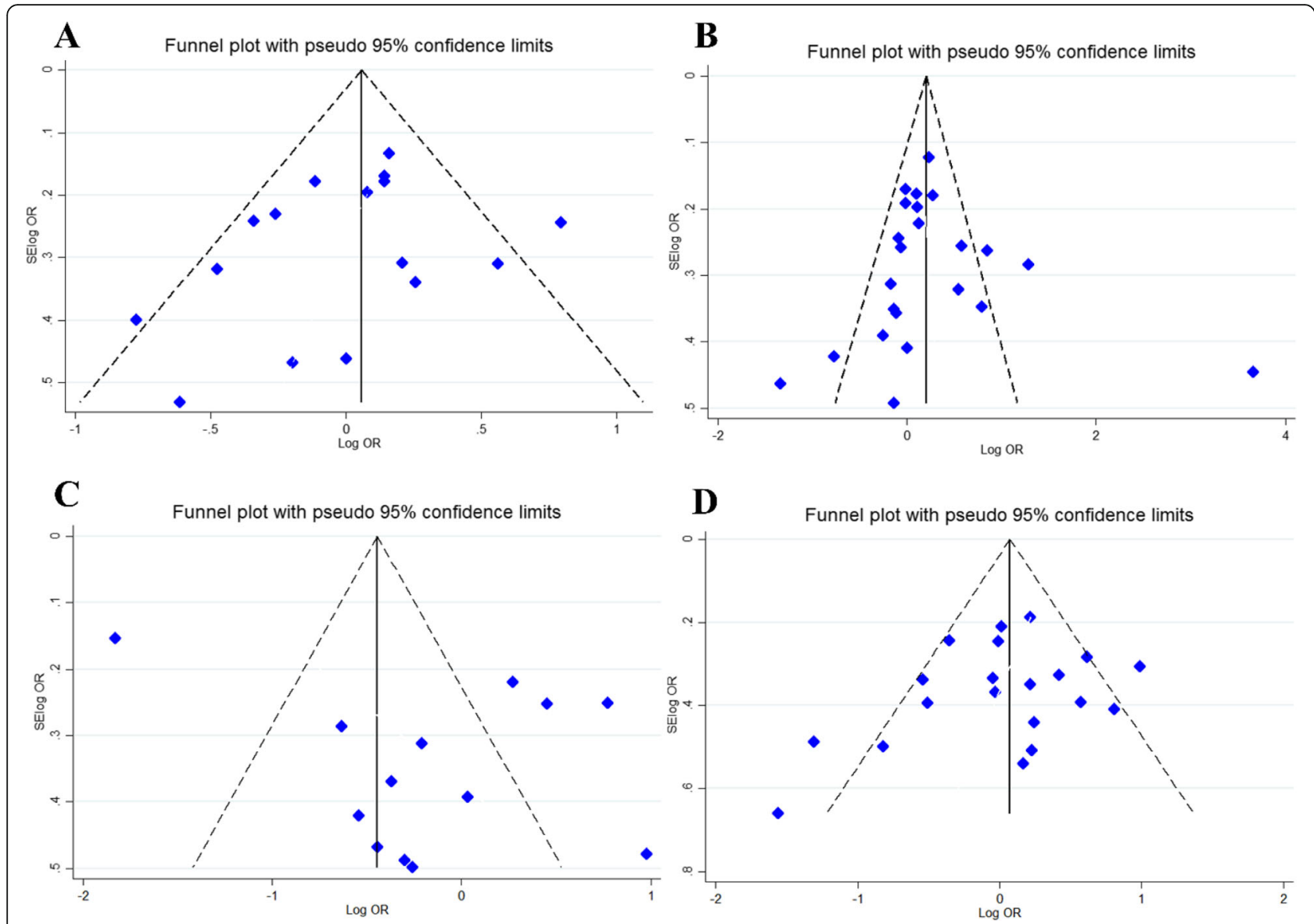

Fig. 4 Begg's funnel plot for publication bias test. a; Dominant Model Fokl .b; Dominant Model Taql. c; Dominant Model Bsml. d; Dominant Model Apal. Each point represents a separate study for the indicated association

Several epidemiological studies have strongly proposed that vitamin D insufficiency and sunshine contributes to MS risk in temperate countries. Vitamin D sufficiency and insufficiency could be a protective and risk factor, respectively, among many other factors, and may be constantly regulating the global MS susceptibility from the mother's pregnancy to adulthood. The main role of vitamin D in MS seems to be immunomodulatory, affecting the different groups of $\mathrm{T}$ and $\mathrm{B}$ cells in the general immune system, however, neurotrophic and neuroprotectant mechanisms could also be applied at the central nervous system (CNS) $[62,63]$. Interestingly, in clinical setting, correction of hypovitaminosis $\mathrm{D}$ through recommending oral D3 supplements resulted in raises in $25(\mathrm{OH}) \mathrm{D}$ levels in serum, which were correlated with reductions in annualized relapse-rate (ARR) in relapsing-remitting MS (RRMS) [64]. The disease activity is generally improved with higher $25(\mathrm{OH}) \mathrm{D}$ level. Rotstein et al.. reported that in MS patients under fingolimod (FTY) therapy, higher $25(\mathrm{OH}) \mathrm{D}$ level was related to longer survival for the combined endpoint and for relapses [65]. A recent randomized clinical trial reveled a potential therapeutic effect of cholecalciferol in RRMS patients with low serum 25OHD level, which already treated with interferon beta-1a [66]. Furthermore, comprehensive systematic review by Dörr et al.. based on many line of data, including preclinical investigations, association studies, epidemiologic data, and animal studies on vitamin D status and disease activity, implies that higher serum level of vitamin D are beneficial in terms of the susceptibility to MS as well as the further course of the disease in patients with established MS [67]. In the earliest phase of disease, lower levels of 25hydroxyvitamin D correlates with higher disease activity, however, lower 25(OH)D3 levels hardly affects patients in terms of clinical presentations, implying that low $25(\mathrm{OH}) \mathrm{D} 3$ concentrations are rather a susceptibility factor for than an outcome of MS; Since the bioavailable vitamin D concentration did not differ between the MS patients and healthy subjects, the main mechanism underlying the association of vitamin D and MS does not seem to be linked with decreased vitamin D bioavailability [68]. Despite all that has been discussed, a meta-analysis by Zheng and colleagues reported that vitamin D had no therapeutic effect on ARR and Expanded Disability Status Scale (EDSS) score in the patients with MS [69]. 


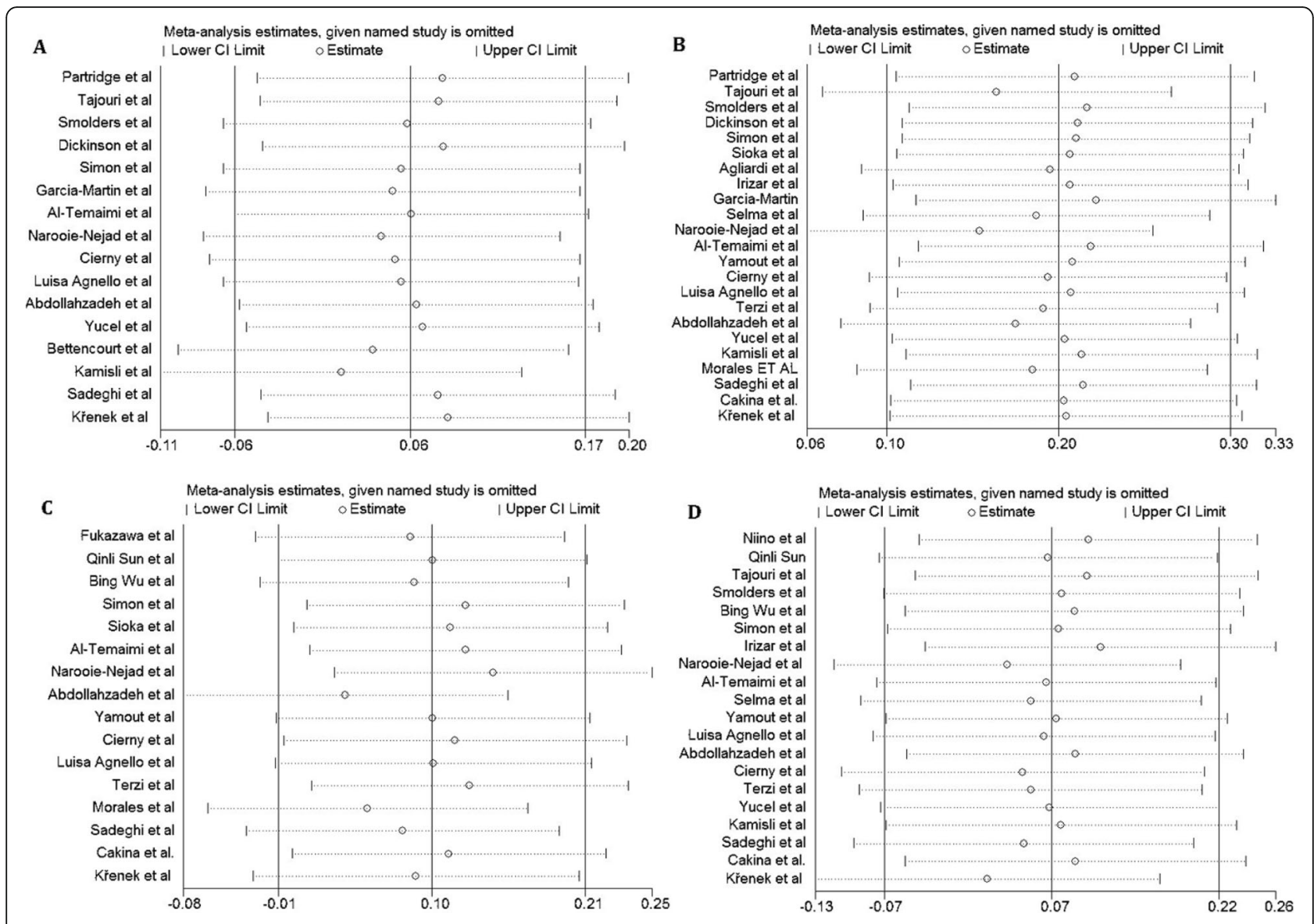

Fig. 5 Sensitivity analysis in present meta-analysis investigates the single nucleotide polymorphisms of Vitamin $D$ Receptor contribute to risk for multiple sclerosis susceptibility (A, Fokl; B, Taql; C, Bsml; D, Apal)

Permutations and combinations of common variants account as a predisposition factors in the etiology of several complex diseases. Variations of DNA sequence like SNPs exert modest biological impacts [11]. Three polymorphisms of VDR gene, including TaqI, ApaI, and BsmI do not influence the structure of VDR protein. Their affect may be associated with alterations in translation efficiency and/or stability of the RNA. On the other hand, the FokI polymorphism has been related to changes in both transcriptional activity and VDR protein structure [70]. The wildtype short transcript of FokI is related with the elevated transcriptional activity [70]. One potential exception is differential effect of the FokI polymorphism on the immune system [16]. Our data suggested that the ApaI polymorphism has a significant functional effect on MS. Furthermore, the TaqI polymorphism was associated with MS risk. However, some other factors that were not examined in the current meta-analysis might affect the TaqI expression. At this point, the expression and function of VDR in transactivating target genes are indicated by environment, genetics, and ethnicity due to its complex interactions [71]. Thus far, three essential environmental risk factors for MS have been determined: vitamin D insufficiency, cigarette smoking, and Epstein-Barr virus infection [72, 73]. Moreover, sun exposure interacts with VDR gene functional variants in childhood to affect MS predisposition.

Some limitations of this meta-analysis should be considered. First, inaccessibility to the original data of the included studies restricted our further assessment of potential interactions, since the interactions between and even various polymorphic region of the same gene may affect the risk. Moreover, this study was solely focused on the articles published in the English language. We detected significant heterogeneity in all of the genetic models, which could be derived by various factors, such as variations in ethnicities. In the current study, ethnicities were Caucasians from Asians, Caucasians from Europe and Australia. Also, heterogeneity may be created by publication year of included studies, which extended between 1999 and 2018. There are several other possible reasons which may be regarded as a source of heterogeneity. Firstly, 
the criteria of MS diagnosis are inconsistent between studies. While some of them employed Poser's criteria, other studies used McDonald's criteria for MS diagnosis. Secondly, gender may act as a potential source for heterogeneity. Although both male and female subjects were enrolled in most studies, two studies were not sex-matched and one study only included women subjects $[35,43,45]$. Thirdly, genotyping methods were not consistent. While half of the included studies used PCR-RFLP, approximately the other half employed TaqMan assay and one study used PCR-SPP. Fourthly, geographical and ethnic factors may also participate in heterogeneity, because studies with the same ethnic source were accompanied in various geographical regions.

The results from the studies examined in this metanalysis should be interpreted with cautious for some reasons. Our findings suggest that, to afford accurate estimates of the relation between VDR polymorphisms and MS risk, several factors should be regarded. Although there are many functional VDR polymorphisms in the promoter region of the VDR gene, only four SNPs in the VDR gene have been evaluated. The interaction of the $M H C$ genes with $V D R$ gene have been demonstrated to be important in MS [74, 75]. Remarkably, various environmental factors may interact with VDR polymorphisms to alter MS susceptibility. The current metaanalysis could not assess all interactions between VDR polymorphisms and study characteristics because of insufficient data from the original publications.

\section{Conclusion}

Taken all together, the current meta-analysis affords a comprehensive investigation of the available information for the association between the VDR polymorphisms and MS susceptibility. This meta-analysis of 30 case-control studies reveled a significant association between TaqI polymorphism and MS susceptibility. In subgroup analysis, BsmI polymorphism was associated with increased risk of MS in Asian populations. In addition, ApaI polymorphism was associated with decreased risk of MS in Asian populations. However, neither in overall population nor in subgroup analysis significant association between Fok1 (rs2228570) polymorphism and MS susceptibility was found. Future large-scale studies on gene-environment and gene-gene interactions are required to estimate related risk factors and assist early diagnosis of patients at high risk for MS.

\section{Supplementary information}

Supplementary information accompanies this paper at https://doi.org/10. 1186/s12883-019-1577-y.

Additional file 1: Figure 1. Forest plot of association between Fok1 gene Polymorphism and MS risk; Dominant model, Recessive model allelic model, ff VS. FF model, Ff vs FF model. Figure 2. Forest plot of pooled odds ratio (OR)) and $95 \%$ confidence interval of individual studies and pooled data for the association between Fok1 polymorphism and MS risk in different ethnicity subgroups and overall populations for Dominant model, Recessive model, allelic model, ff VS. FF model, Ff vs FF model. Figure 3. Forest plot of association between Taq1 gene Polymorphism and MS risk; Dominant model, Recessive model, allelic model, tt VS. TT model, Tt vs TT model. Figure 4. Forest plot of pooled odds ratio (OR)) and 95\% confidence interval of individual studies and pooled data for the association between Taq1 polymorphism and MS risk in different ethnicity subgroups and overall populations for Dominant model, Recessive model, allelic model, tt vs TT model, Tt vs TT model. Figure 5. Forest plot of association between Bsm1 gene Polymorphism and MS risk; Dominant model, Recessive model, allelic model, bb VS. BB model, Bb vs BB model. Figure 6. Forest plot of pooled odds ratio (OR)) and $95 \%$ confidence interval of individual studies and pooled data for the association between Bsm1polymorphism and MS risk in different ethnicity subgroups and overall populations for Dominant model, Recessive model allelic model, bb VS. BB model, Bb vs BB model. Figure 7. Forest plot of association between Apa1 gene Polymorphism and MS risk; Dominant model, Recessive model, allelic model, aa VS. AA model, Aa vs AA model. Figure 8. Forest plot of pooled odds ratio (OR)) and $95 \%$ confidence interval of individual studies and pooled data for the association between Apa1 polymorphism and MS risk in different ethnicity subgroups and overall populations for Dominant model, Recessive model, allelic model, aa VS. AA model, Aa vs AA model.

\section{Abbreviations}

Cl: Confidence Interval; CNS: Central nervous system; HLA: Human leukocyte antigen; LD: Linkage disequilibrium; MHC-II: Major histocompatibility complex II; MS: Multiple sclerosis; OR: Odd Ratio; PCR-SPP: Polymerase chain reaction Sequence Specific; PRISMA: Preferred Reporting Items for Systematic reviews and Meta-Analyses; RFLP: Restriction fragment length polymorphisms; SLE: Systemic lupus erythematosus; SNP: Single Nucleotide Polymorphisms; T1D: Type 1 diabetes; UTR: Untranslated region; VDR: Vitamin D receptor polymorphism

\section{Acknowledgements}

We would like to thank Dr.Saeid Aslani for his valuable comments that greatly improved the manuscript.

\section{Authors' contributions}

Conceived and designed the experiments: DI and BR. Extracted data: DI and MA. Performed the data analysis: BR. Writing original draft: RR and BR. All authors read and approved the final manuscript.

\section{Authors' information}

Ramazan Rezaei is an associate researcher of the department of Immunology at Shahid Beheshti University of Medical Sciences, Tehran, Iran. His research focuses on autoimmune diseases, Immunogenetics, and Immunotherapy, with a particular interest in the Manipulation of Tumor deriver exosomes as a therapeutic vaccine in colorectal cancer.

\section{Funding}

This research did not receive any specific grant from funding agencies in the public, commercial, or not-for-profit sector.

\section{Availability of data and materials}

The datasets used and/or analysed during the current study are available from the corresponding author on reasonable request.

Ethics approval and consent to participate

This study has been approved by ethic committee of Tehran University of Medical Sciences.

Consent for publication

Not applicable.

Competing interests

The authors declare that they have no competing interests. 


\section{Author details}

'Department of Immunology, School of Public Health, Tehran University of Medical Sciences(TUMS), Tehran, Iran. ${ }^{2}$ Department of Hematology and Blood Banking, School of Medicine, Tarbiat modares university (TMU), Tehran, Iran. ${ }^{3}$ Immunology Research Center, Tabriz University of Medical Sciences, Tabriz, Iran. ${ }^{4}$ Student Research Committee, Tabriz University of Medical Sciences, Tabriz, Iran. ${ }^{5}$ Department of Immunology, Faculty of Medicine, Iran University of Medical Sciences, Tehran, Iran. ${ }^{6}$ Department of Immunology, School of Medicine, Shahid Beheshti University of Medical Sciences, Tehran 14194, Iran.

Received: 24 October 2019 Accepted: 16 December 2019 Published online: 26 December 2019

\section{References}

1. Imani $D$, et al. Association of nod-like receptor protein-3 single nucleotide gene polymorphisms and expression with the susceptibility to relapsingremitting multiple sclerosis. Int J Immunogenet. 2018;45(6):329-36.

2. Emamnejad $\mathrm{R}$, et al. Circulating mesenchymal stem cells, stromal derived factor (SDF)-1 and IP-10 levels increased in clinically active multiple sclerosis patients but not in clinically stable patients treated with beta interferon. Mult Scler Relat Disord. 2019;35:233-8.

3. Longo DL, Reich DS, Lucchinetti CF, Calabresi PA. N Engl J Med. 2018;378: 169-80.

4. Krieger SC, et al. The topographical model of multiple sclerosis: a dynamic visualization of disease course. Neurology-Neuroimmunol Neuroinflamm. 2016;3(5):e279.

5. McFarland HF, Martin R. Multiple sclerosis: a complicated picture of autoimmunity. Nat Immunol. 2007;8(9):913.

6. Adorini L, Penna G. Control of autoimmune diseases by the vitamin D endocrine system. Nat Rev Rheumatol. 2008;4(8):404.

7. DeLuca HF. Overview of general physiologic features and functions of vitamin D. Am J Clin Nutr. 2004:80(6):1689S-96S.

8. Gorman S, et al. Topically applied 1, 25-dihydroxyvitamin D3 enhances the suppressive activity of CD4+ CD25+ cells in the draining lymph nodes. J Immunol. 2007;179(9):6273-83.

9. Smolders J, et al. Association of vitamin D metabolite levels with relapse rate and disability in multiple sclerosis. Mult Scler J. 2008;14(9):1220-4.

10. Runia TF, et al. Lower serum vitamin D levels are associated with a higher relapse risk in multiple sclerosis. Neurol. 2012;79(3):261-6.

11. Uitterlinden $A G$, et al. Genetics and biology of vitamin $D$ receptor polymorphisms. Gene. 2004;338(2):143-56.

12. Zmuda JM, Cauley JA, Ferrell RE. Molecular epidemiology of vitamin D receptor gene variants. Epidemiol Rev. 2000;22(2):203-17.

13. Makoui $\mathrm{MH}$, et al. Vitamin D receptor gene polymorphism and susceptibility to asthma: meta-analysis based on 17 case-control studies. Ann Allergy Asthma Immunol. 2019;124(1):57-69.

14. Ručević I, et al. Vitamin D endocrine system and psoriasis vulgaris-review of the literature. Acta Dermatovenerol Croat. 2009;17(3):0-0.

15. Saccone $D$, Asani F, Bornman L. Regulation of the vitamin D receptor gene by environment, genetics and epigenetics. Gene. 2015;561(2):171-80.

16. van Etten $\mathrm{E}$, et al. The vitamin $\mathrm{D}$ receptor gene Fokl polymorphism: functional impact on the immune system. Eur J Immunol. 2007;37(2):395-405.

17. Tajouri $L$, et al. Variation in the vitamin $D$ receptor gene is associated with multiple sclerosis in an Australian population. J Neurogenet. 2005; 19(1):25-38.

18. Al-Temaimi RA, et al. The association of vitamin D receptor polymorphisms with multiple sclerosis in a case-control study from Kuwait. PLoS One. 2015; 10(11):e0142265

19. Narooie-Nejad M, et al. Positive association of vitamin D receptor gene variations with multiple sclerosis in South East Iranian population. BioMed Res Int. 2015;44(3):174-85.

20. Ben-Selma W, et al. Age-and gender-specific effects on VDR gene polymorphisms and risk of the development of multiple sclerosis in Tunisians: a preliminary study. Int J Immunogenet. 2015;42(3):174-81.

21. Čierny $D$, et al. Fokl vitamin $D$ receptor gene polymorphism in association with multiple sclerosis risk and disability progression in Slovaks. Neurol Res. 2015;37(4):301-8.

22. Sioka $\mathrm{C}$, et al. Vitamin D receptor gene polymorphisms in multiple sclerosis patients in Northwest Greece. J Negat Results Biomed. 2011;10(1):3.
23. Huang J, Xie Z-F. Polymorphisms in the vitamin D receptor gene and multiple sclerosis risk: a meta-analysis of case-control studies. J Neurol Sci. 2012;313(1-2):79-85.

24. García-Martín E, et al. Vitamin D3 receptor (VDR) gene rs2228570 (Fok1) and rs731236 (Taq1) variants are not associated with the risk for multiple sclerosis: results of a new study and a meta-analysis. PLoS One. 2013;8(6):e65487.

25. Zhang Y-J, et al. Association between VDR polymorphisms and multiple sclerosis: systematic review and updated meta-analysis of case-control studies. Neurol Sci. 2018;39(2):225-34.

26. Tizaoui $\mathrm{K}$, et al. Association between vitamin $\mathrm{D}$ receptor polymorphisms and multiple sclerosis: systematic review and meta-analysis of case-control studies. Cell Mol Immunol. 2015;12(2):243.

27. Moher D, et al. Preferred reporting items for systematic reviews and metaanalyses: the PRISMA statement. Ann Intern Med. 2009;151(4):264-9.

28. Stang A. Critical evaluation of the Newcastle-Ottawa scale for the assessment of the quality of nonrandomized studies in meta-analyses. Eur J Epidemiol. 2010;25(9):603-5.

29. Huedo-Medina TB, et al. Assessing heterogeneity in meta-analysis: Q statistic or 12 index? Psychol Methods. 2006;11(2):193.

30. Mantel N, Haenszel W. Statistical aspects of the analysis of data from retrospective studies of disease. J Natl Cancer Inst. 1959;22(4):719-48.

31. DerSimonian, R. and N. Laird, Meta-analysis in clinical trials control Clin trials 7: 177-188. Find this article online, 1986.

32. Egger $M$, et al. Bias in meta-analysis detected by a simple, graphical test. BMJ. 1997;315(7109):629-34.

33. Begg CB, Mazumdar M. Operating characteristics of a rank correlation test for publication bias. Biometrics. 1994:1088-101.

34. Partridge J, et al. Susceptibility and outcome in MS: associations with polymorphisms in pigmentation-related genes. Neurol. 2004;62(12):2323-5.

35. Smolders J, et al. Fok-I vitamin D receptor gene polymorphism (rs10735810) and vitamin D metabolism in multiple sclerosis. J Neuroimmunol. 2009; 207(1-2):117-21.

36. Agnello $L$, et al. Vitamin D receptor polymorphisms and 25-hydroxyvitamin D in a group of Sicilian multiple sclerosis patients. Neurol Sci. 2016;37(2):261-7.

37. Yucel FE, et al. Analysis of vitamin D receptor polymorphisms in patients with familial multiple sclerosis. Med Arch. 2018;72(1):58.

38. Bettencourt $\mathrm{A}$, et al. The vitamin $\mathrm{D}$ receptor gene Fokl polymorphism and multiple sclerosis in a northern Portuguese population. J Neuroimmunol. 2017;309:34-7

39. Kamisli $\mathrm{O}$, et al. The association between vitamin $\mathrm{D}$ receptor polymorphisms and multiple sclerosis in a Turkish population. Mult Scler Relat Dis. 2018;20:78-81.

40. Krenek P, et al. The Impact of Five VDR Polymorphisms on Multiple Sclerosis Risk and Progression: a Case-Control and Genotype-Phenotype Study. J Mol Neurosci. 2018:1-8.

41. Abdollahzadeh $\mathrm{R}$, et al. Predisposing role of vitamin $\mathrm{D}$ receptor (VDR) polymorphisms in the development of multiple sclerosis: a case-control study. J Neurol Sci. 2016;367:148-51.

42. SADEGHI A, et al. The Bsml, Fokl, Apal and Taql Polymorphisms in Vitamin D Receptor Gene in Iranian Multiple Sclerosis Patients: A Case-Control Study. J iranian clin Res. 2015;3:28-32.

43. Simon KC, et al. Polymorphisms in vitamin D metabolism related genes and risk of multiple sclerosis. Mult Scler J. 2010;16(2):133-8.

44. Dickinson $\mathrm{J}$, et al. Past environmental sun exposure and risk of multiple sclerosis: a role for the $\mathrm{Cdx}-2$ vitamin $\mathrm{D}$ receptor variant in this interaction. Mult Scler J. 2009:15(5):563-70.

45. Smolders J, et al. Association study on two vitamin D receptor gene polymorphisms and vitamin D metabolites in multiple sclerosis. Ann N Y Acad Sci. 2009;1173(1):515-20.

46. Irizar $\mathrm{H}$, et al. HLA-DRB1* 15: 01 and multiple sclerosis: a female association? Mult Scler J. 2012;18(5):569-77.

47. Agliardi C, et al. Vitamin D receptor (VDR) gene SNPs influence VDR expression and modulate protection from multiple sclerosis in HLA-DRB1* 15-positive individuals. Brain Behav Immun. 2011;25(7):1460-7.

48. Cakina $\mathrm{S}$, et al. Vitamin D receptor gene polymorphisms in multiple sclerosis disease: a case-control study. Rev Romana De Med De Lab. 2018;26(4):489-95.

49. Terzi, M., et al. Vitamin D Receptor Gene Polymorphism in Patients with Multiple Sclerosis. in MULTIPLE SCLEROSIS JOURNAL. 2018. SAGE PUBLICATIONS LTD 1 OLIVERS YARD, 55 CITY ROAD, LONDON EC1Y 1SP ENGLAND.2018. 24(7) pp. 61-62.

50. Narooie-Nejad $\mathrm{M}$, et al. Vitamin $\mathrm{D}$ receptor gene polymorphism and the risk of multiple sclerosis in south eastern of Iran. J Mol Neurosci. 2015;56(3):572-6. 
51. Yamout B, et al. Vitamin D receptor biochemical and genetic profiling and HLA-class II genotyping among Lebanese with multiple sclerosis-a pilot study. J Neuroimmunol. 2016;293:59-64.

52. Bermúdez-Morales $\mathrm{VH}$, et al. Vitamin $\mathrm{D}$ receptor gene polymorphisms are associated with multiple sclerosis in Mexican adults. J Neuroimmunol. 2017; 306:20-4.

53. Fukazawa $T$, et al. Association of vitamin $D$ receptor gene polymorphism with multiple sclerosis in Japanese. J Neurol Sci. 1999; 166(1):47-52.

54. Bing WHL, Tao W, et al. Association of vitamin D receptor gene polymorphism with multiple sclerosis. Henan Med Res. 2009:49(24):86-7.

55. Qinlin SRX, Yinhua W, et al. Association of vitamin D receptor gene polymorphism with multiple sclerosis. Chin J Tissue Eng Res. 2004

56. Niino $\mathrm{M}$, et al. Vitamin $\mathrm{D}$ receptor gene polymorphism in multiple sclerosis and the association with HLA class II alleles. J Neurol Sci. 2000; 177(1):65-71.

57. Feng $\mathrm{M}$, et al. Polymorphisms in the vitamin $\mathrm{D}$ receptor gene and risk of autoimmune thyroid diseases: a meta-analysis. Endocrine. 2013;43(2):318326.

58. Mao S, Huang S. Association between vitamin D receptor gene Bsml, Fokl, Apal and Taql polymorphisms and the risk of systemic lupus erythematosus: a meta-analysis. Rheumatol Int. 2014;34(3):381-8.

59. Tizaoui K, Hamzaoui K. Association between VDR polymorphisms and rheumatoid arthritis disease: systematic review and updated meta-analysis of case-control studies. Immunobiol. 2015;220(6):807-16.

60. Wang G, Kuanfeng X, Yang T. Associations between polymorphisms of vitamin $\mathrm{D}$ receptor gene and type 1 diabetes susceptibility: a meta-analysis. Chin J Diab. 2015;2:110-4.

61. Morán-Auth Y, Penna-Martinez M, Badenhoop K. VDR Fokl polymorphism is associated with a reduced T-helper cell population under vitamin D stimulation in type 1 diabetes patients. J Steroid Biochem Mol Biol. 2015; 148:184-6.

62. Pierrot-Deseilligny C, Souberbielle J-C. Vitamin D and multiple sclerosis: an update. Mult Scler Relat Dis. 2017;14:35-45.

63. Koduah P, Paul F, Dörr J-M. Vitamin D in the prevention, prediction and treatment of neurodegenerative and neuroinflammatory diseases. Epma J. 2017:8(4):313-25.

64. Laursen JH, et al. Vitamin D supplementation reduces relapse rate in relapsing-remitting multiple sclerosis patients treated with natalizumab. Mult Scler Relat Dis. 2016;10:169-73.

65. Rotstein DL, et al. Effect of vitamin D on MS activity by disease-modifying therapy class. Neurol-Neuroimmunol Neuroinflamm. 2015;2(6):e167.

66. Camu W, et al. Cholecalciferol in relapsing-remitting MS: a randomized clinical trial (CHOLINE). Neurol-Neuroimmunol Neuroinflamm. 2019;6(5):e597.

67. Dörr J, Döring A, Paul F. Can we prevent or treat multiple sclerosis by individualised vitamin D supply? Epma J. 2013:4(1):4.

68. Behrens JR, et al. Low 25-hydroxyvitamin D, but not the bioavailable fraction of 25-hydroxyvitamin $D$, is a risk factor for multiple sclerosis. Eur J Neurol. 2016;23(1):62-7.

69. Zheng $C$, et al. The efficacy of vitamin $D$ in multiple sclerosis: a metaanalysis. Mult Scler Relat Dis. 2018;23:56-61.

70. Jurutka PW, et al. The polymorphic $N$ terminus in human vitamin D receptor isoforms influences transcriptional activity by modulating interaction with transcription factor IIB. Mol Endocrinol. 2000;14(3):401-20.

71. O'Gorman C, Lucas R, Taylor B. Environmental risk factors for multiple sclerosis: a review with a focus on molecular mechanisms. Int J Mol Sci. 2012;13(9):11718-52.

72. Pierrot-Deseilligny C, Souberbielle J-C. Contribution of vitamin D insufficiency to the pathogenesis of multiple sclerosis. Ther Adv Neurol Disord. 2013;6(2):81-116.

73. Canbay C. The essential environmental cause of multiple sclerosis disease Prog Electromagn Res. 2010;101:375-91.

74. Handunnetthi L, Ramagopalan SV, Ebers GC. Multiple sclerosis, vitamin D, and HLA-DRB1* 15. Neurol. 2010;74(23):1905-10

75. Ramagopalan SV, et al. Expression of the multiple sclerosis-associated MHC class II allele HLA-DRB1* 1501 is regulated by vitamin D. PLoS Genet. 2009; 5(2):e1000369.

\section{Publisher's Note}

Springer Nature remains neutral with regard to jurisdictional claims in published maps and institutional affiliations.

Ready to submit your research? Choose BMC and benefit from:

- fast, convenient online submission

- thorough peer review by experienced researchers in your field

- rapid publication on acceptance

- support for research data, including large and complex data types

- gold Open Access which fosters wider collaboration and increased citations

- maximum visibility for your research: over $100 \mathrm{M}$ website views per year

At BMC, research is always in progress.

Learn more biomedcentral.com/submissions 